\title{
Routh reduction for singular Lagrangians
}

\author{
Bavo Langerock* \\ Sint-Lucas school of Architecture \\ B-9000 Ghent, Belgium
}

\author{
Marco Castrillón López \\ ICMAT (CSIC-UAM-UC3M-UCM), Dept. Geometría y Topología \\ Facultad de Matemáticas \\ Universidad Complutense de Madrid \\ 28040 Madrid, Spain
}

May 30, 2018

\begin{abstract}
This paper concerns the Routh reduction procedure for Lagrangians systems with symmetry. It differs from the existing results on geometric Routh reduction in the fact that no regularity conditions on either the Lagrangian $L$ or the momentum map $J_{L}$ are required apart from the momentum being a regular value of $J_{L}$. The main results of this paper are: the description of a general Routh reduction procedure that preserves the Euler-Lagrange nature of the original system and the presentation of a presymplectic framework for Routh reduced systems. In addition, we provide a detailed description and interpretation of the Euler-Lagrange equations for the reduced system. The proposed procedure includes Lagrangian systems with a non-positively definite kinetic energy metric.

MSC2000: Primary 70H33. Secondary 70G65, 70H03, 53D20.
\end{abstract}

Keywords: Constrained Lagrangian systems; momentum map; reduction; Routhian; singular Lagrangians; symmetry; symplectic form.

\section{Contents}

1 Introduction 2

2 Lagrangian systems on fibred manifolds 4

2.1 Gyroscopic forces . . . . . . . . . . . . . . . . . . . . . 6

2.2 Regular configuration constraints . . . . . . . . . . . . . . 7

2.3 Linear constraints and Lagrangian multipliers . . . . . . . . . . . . . . . . 8

2.4 Connections . . . . . . . . . . . . . . . . . . . . . . . . . . . . 9

* BL is an honorary postdoctoral researcher at the Department of Mathematics of Ghent Univeristy and associate academic staff at the Department of Mathematics of KULeuven. 
3 Lagrangian systems with symmetry 10

3.1 General constructions on the quotient spaces . . . . . . . . . . . . . 12

3.2 The regular case . . . . . . . . . . . . . . . . . . 13

3.3 The reduced form induced by the connection $\ldots \ldots \ldots \ldots \ldots$

3.4 Variations . . . . . . . . . . . . . . . . . . . . 17

3.5 Lagrange-Poincaré reduction . . . . . . . . . . . . . . . . . 18

4 The Routhian reduction scheme 19

4.1 Remarks on Routh reduction . . . . . . . . . . . . . . . . . 223

5 Reconstruction 25

6 Routhian reduced systems: standard cases and examples 26

6.1 The regular case . . . . . . . . . . . . . . . . . . . . . 26

6.1.1 The (pre)-symplectic nature of classical Routhian reduction . . . . . . . 28

6.1 .2 The modified Tippe Top . . . . . . . . . . . . . . . 28

6.1 .3 The free rigid body . . . . . . . . . . . . . . . . . 29

6.2 Lagrangian systems subjected to magnetic forces . . . . . . . . . . . . . . 31

6.2 .1 The heavy top in a magnetic field . . . . . . . . . . . . . . . . 31

6.3 Lagrangians linear in the symmetry generators . . . . . . . . . . . . . . 32

$6.3 .1 \quad$ Lightlike Killing vectors . . . . . . . . . . . . . . . . . . . . 32

\section{Introduction}

In geometric accounts to Routh reduction [2, 4, 9, 15] it is custom to first consider the restriction of the dynamics associated to a Lagrangian system with symmetry to the level set of the momentum map $J_{L}^{-1}(\mu)$ and then reduce to the quotient manifold under the action of the isotropy group $G_{\mu}$. Because this procedure is very similar to symplectic or Marsden-Weinstein reduction, one often states that Routh reduction is the Lagrangian analogue of cotangent bundle reduction (see e.g. 9, 15). Another essential ingredient in the work on Routh reduction is the existence of a regularity condition on the momentum map $J_{L}$ : it guarantees that the manifold $J_{L}^{-1}(\mu) / G_{\mu}$ is diffeomorphic to $T(Q / G) \times Q / G_{\mu}$. Roughly said, this condition provides the manifold $J_{L}^{-1}(\mu) / G_{\mu}$ with a tangent bundle structure, an essential feature to reinterpret the reduced dynamics as being Euler-Lagrange equations. We say that Lagrangians satisfying this regularity condition are $G$ regular. For example, the classical $T-V$ Lagrangians fit in this context. Unfortunately, there are many Lagrangians where this procedure can not be carried out. This is the case for singular Lagrangians, though there are also instances of regular Lagrangians where it is impossible to perform Routhian reduction in this sense. A simple one is the Lagrangian $L\left(q^{1}, q^{2}, \dot{q}^{1}, \dot{q}^{2}\right)=$ $\left(\dot{q}^{1}\right)^{2}+\dot{q}^{1} \dot{q}^{2}-V\left(q^{1}\right)$ defined in $Q=\mathbb{R}^{2}$ and symmetric with respect to the action of the group $G=\mathbb{R}$ of translations along the $q^{2}$ coordinate (using the old language in Mechanics, $q^{2}$ is a cyclic coordinate). The momentum map is $J_{L}=\dot{q}^{1}$. For a frozen value $\mu=\dot{q}^{1}$ of $J_{L}$, the quotient 


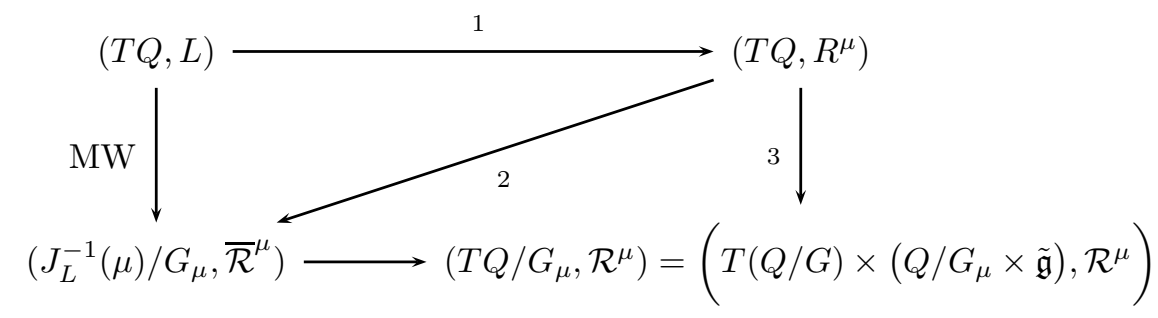

Figure 1: Schematic drawing of reduction on a tangent bundle

$J_{L}^{-1}(\mu) / G_{\mu}$ can not be identified with $T(Q / G) \times Q / G_{\mu}=T(Q / G)$ as the coordinates of the quotient space $J_{L}^{-1}(\mu) / G_{\mu}$ are precisely $\left(q^{1}, \dot{q}^{2}\right)$ whereas the coordinates of $T(Q / G)$ are $\left(q^{1}, \dot{q}^{1}\right)$. See 33.2 below. The main result of this paper is the generalization of Routh reduction to a reduction technique that holds for arbitrary Lagrangians with the only requirement of $\mu$ being a regular value of $J_{L}$. The price paid for that is that the reduction process will be carried out in the entire space $T Q$ and not only on $J_{L}^{-1}(\mu)$. The fact that the reduction takes place in the whole space does not mean that we loose control of the momentum, at the contrary, the reduction process will keep track of the value of $J_{L}$ in the same spirit of the classical Routh reduction.

In order to relate the presented reduction technique to existing results on geometric Routh reduction, we first mention that the correspondence between Routh reduction and Marsden-Weinstein reduction holds at a much more fundamental level: Routh-reduction is Marsden-Weinstein reduction applied to the Poincaré-Cartan symplectic structure on the tangent bundle (see [12]). This correspondence was then used to generalize Routh reduction to Lagrangians that are quasiinvariant (up to a total time derivative).

Here we wish to generalize Routh reduction from a different point of view. In classical treatments on Routh reduction of a Lagrangian system with cyclic coordinates, the reduced system is again a Lagrangian system with a new Lagrangian which is called the Routhian. It is this observation what we take as a starting point in this paper: Routh reduction is a reduction technique that preserves the Euler-Lagrange nature of the original system.

The different steps in the proposed reduction are best illustrated by means of the schematic diagram in Fig. 1. For that purpose, we shall denote for now a Lagrangian system as a couple $(T Q, L), Q$ being the configuration manifold and $L$ a function on $T Q$ called the Lagrangian. The first step is to consider an equivalent Lagrangian system on $Q$, with Lagrangian $R^{\mu}$. This new Lagrangian has the property that solutions of the Euler-Lagrange equations of $(T Q, L)$ with momentum $\mu$ are solutions to $\left(T Q, R^{\mu}\right)$ with momentum 0 . For the sake of completeness we mention here that $\left(T Q, R^{\mu}\right)$ is not conservative and additional force terms should be taken into account; and that the function $R^{\mu}$ is only $G_{\mu}$ invariant but, as will become clear, we can make sense to a momentum map of $R^{\mu}$ taking values in $\mathfrak{g}^{*}$, the dual of the Lie-algebra of $G$. This step is not new and was carried out, for instance, in 9 . The Routh reduction technique described in [9] is schematically presented in Fig. 1 by the arrows 1 and 2, followed in this order.

We shall follow a different path: in our approach the second step is to perform a reduction on the system $\left(T Q, R^{\mu}\right)$ that is similar to Lagrange-Poincaré reduction, i.e. we reduce the system to $\left(T Q / G_{\mu}, \mathcal{R}^{\mu}\right)$, where $\mathcal{R}^{\mu}$ is the quotient of $R^{\mu}$ (represented by arrow 3 in the above diagram). We show that this step, in contrast to general Lagrange-Poincaré reduction, preserves the EulerLagrange nature of the original system. To explain this statement in more detail, we mention here that $T Q / G_{\mu}$ can be identified with the fibred product of three bundles over $Q / G: T(Q / G)$, $Q / G_{\mu}$ and $\tilde{\mathfrak{g}}$ (the latter being the bundle associated to $\mathfrak{g}$ ). Roughly said, the Routhian $\mathcal{R}^{\mu}$ 
depends on velocities $v_{x}$ in $T(Q / G)$ and on points $y$ and $\tilde{\xi}$ in the fibres of $Q / G_{\mu}$ and $\tilde{\mathfrak{g}}$ above $x$ respectively. When we say that $\mathcal{R}^{\mu}\left(v_{x}, y, \tilde{\xi}\right)$ is a Lagrangian, we understand that the variables $y$ and $\tilde{\xi}$ are to be interpreted as configuration coordinates. This might be surprising, since $\tilde{\xi}$ is the projection of the vertical part of a velocity upstairs, i.e. a tangent vector in $T Q$.

It is immediately clear now that the Lagrangian $\mathcal{R}^{\mu}$ is singular, i.e. it does not depend on the velocities of the variables $y$ and $\tilde{\xi}$. This is another price we pay for keeping the EulerLagrange nature of the system: we have to work with singular Lagrangians. But the singularity of these systems is of a specific type, which we call throughout this paper intrinsically constrained Lagrangian systems. They are studied in detail in Section 2. It is well known that singular Lagrangians contain constraints on the solutions to the associated Euler-Lagrange equations. It is shown that one of these intrinsic constraints embedded in the system $\left(T Q / G_{\mu}, \mathcal{R}^{\mu}\right)$ is a reduced version of the fixed momentum condition $J_{L}=\mu$. As a result of this Lagrangian interpretation of the reduced system $\left(T Q / G_{\mu}, \mathcal{R}^{\mu}\right)$, we shall define a presymplectic formulation and, in the most general case, one can apply the presymplectic constraint algorithm [6, 7, 8, to find solutions to the Euler-Lagrange equations.

Depending on the nature of these constraints we describe in more detail two distinct cases where this reduced singular Lagrangian system is equivalent to a variational problem on the space $J_{L}^{-1}(\mu) / G_{\mu}$; the first case leads to standard Routh reduction and the second case is new and, for instance, is applicable to geodesics in general relativity where the metric is invariant under a lightlike vector field.

Finally, we wish to mention that besides the importance of Routh reduction for Mechanical systems by itself, the special features of this process may shed light to other situations. In particular, this might be the case for Field Theories. The covariant reduction of these Lagrangian systems under the action of a group of symmetries has been developed in recent years (see for example [14]), though the Hamiltonian counterpart is much less explored. A generalization of Routh techniques to this setting could be of much interest to tackle some of the difficulties encountered in this context (for instance, the lack of meaning of the notion of fixing the value of the momentum map).

Throughout this paper manifolds are always assumed to be smooth finite dimensional (Hausdorff, second countable, $C^{\infty}$ ) and smooth always means of class $C^{\infty}$. We will often consider the pullback of a bundle, a function, a one- or two-form along a map $f: M \rightarrow N$ between two manifolds. To reduce the notational complexity in this paper, we sometimes denote the pull-backed object with the same symbol as the object itself. It should be clear from the context what is meant. Given two bundles $\pi_{1}: M_{1} \rightarrow N$ and $\pi_{2}: M_{2} \rightarrow N$ over a manifold $N$, then we often consider the fibred product bundle $M_{1} \times_{N} M_{2}$, or simply $M_{1} \times M_{2}$ when no confusion is possible. Elements in such a fibred product are denoted as a couple $\left(m_{1}, m_{2}\right)$, with $m_{1} \in M_{1}$ and $m_{2} \in M_{2}$ such that $\pi_{1}\left(m_{1}\right)=\pi_{2}\left(m_{2}\right)$. Finally, velocities at a point $m \in M$ are typically denoted by $v_{m}, w_{m} \in T_{m} M$. When we consider the tangent vector to a curve $m: I \rightarrow M$ at a time $t$, we shall write $\dot{m}(t)$ for the corresponding curve in $T M$.

\section{Lagrangian systems on fibred manifolds}

We start with recalling some standard concepts in Lagrangian mechanics. Assume that $L$ is a Lagrangian function defined on the tangent space of a manifold $M$.

Definition $1 A$ Lagrangian system is a triple $(M, L, F)$ with $L$ a function on $T M$ and $F$ a co-vector valued function $T M$, i.e. $F: T M \rightarrow T^{*} M$, fibred over the identity. The map $F$ is 
called the force term of the Lagrangian system. A Lagrangian system $(M, L, F)$ is said to be conservative if $F=0$.

In the above notion of conservative systems we assume that conservative forces are always taken into account by means of their potential energy in the Lagrangian function $L$.

Definition 2 A curve $m: I=[a, b] \rightarrow M$ is said to be a critical curve for the Lagrangian system $(M, L, F)$ if for arbitrary variations $\delta m$ with fixed endpoints $\delta m(a, b)=0$,

$$
\delta \int_{I} L(\dot{m}(t)) d t+\int_{I}\langle F(\dot{m}(t)), \delta m(t)\rangle d t=0
$$

holds.

It is well-known that a critical curve satisfies

$$
\mathcal{E} \mathcal{L}(L)(\ddot{m}(t))+F(\dot{m}(t))=0,
$$

with $\mathcal{E} \mathcal{L}$ the Euler-Lagrange operator $\mathcal{E} \mathcal{L}(L): T^{(2)} M \rightarrow T^{*} M$. When expressed in a local coordinate system $\left(X^{i}\right)$ on $M$, we get

$$
\mathcal{E} \mathcal{L}(L)(\ddot{m}(t))=\left(\frac{\partial L}{\partial X^{i}}(X, \dot{X})-\frac{d}{d t}\left(\frac{\partial L}{\partial \dot{X}^{i}}\right)(X, \dot{X}, \ddot{X})\right) d X^{i} .
$$

Thus a critical curve satisfies

$$
\frac{d}{d t}\left(\frac{\partial L}{\partial \dot{X}^{i}}\right)-\frac{\partial L}{\partial X^{i}}=F_{i}, \text { for } i=1, \ldots, \operatorname{dim} M
$$

We now assume that the manifold $M$ is fibred over $N$, i.e. there is a bundle map $\pi: M \rightarrow N$. We denote the kernel of $T \pi$ by $V \pi \subset T M$ and is called the bundle of vertical tangent vectors. A cotangent vector $\alpha_{m} \in T_{m}^{*} M$ can be restricted to $V_{m} \pi$, and such a restriction will be denoted by $\alpha_{m}^{v}: V_{m} \pi \rightarrow \mathbb{R}$. Moreover, we denote the product bundle $T N \times_{N} M$ by $T_{M} N$. Elements in this bundle are denoted by $\left(v_{n}, m\right)$, where $v_{n} \in T_{n} N$ and $m \in M_{n}=\pi^{-1}(n)$. The map $\left(T \pi, \tau_{M}\right): T M \rightarrow T_{M} N ; v_{m} \mapsto\left(T \pi\left(v_{m}\right), m\right)$ is denoted by $p_{1}$.

Definition 3 An intrinsically constrained Lagrangian system is a triple $(\pi: M \rightarrow N, L, F)$, with $L$ a function on $T_{M} N$ and $F$ the force term $F: T M \rightarrow T^{*} M$ fibred over the identity on $M$. A curve $m(t) \in M$ is said to be a critical curve of the intrinsically constrained Lagrangian system if it is a critical curve of the associated Lagrangian system $\left(M, p_{1}^{*} L, F\right)$.

It should be clear that the Lagrangian system $\left(M, p_{1}^{*} L, F\right)$ is singular since $p_{1}^{*} L$ is independent of the velocities in the fibre coordinates. We now focus on the Euler-Lagrange equations for the Lagrangian system $\left(M, p_{1}^{*} L, F\right)$ associated to the intrinsically constrained system $(\pi: M \rightarrow$ $N, L, F$ ) (from now on, we denote $L$ and $p_{1}^{*} L$ by the same symbol). For that purpose, let $\left(x^{i}, y^{a}\right)$ be a coordinate system on $M$ that is adapted to the fibration, i.e. $\left(x^{i} ; i=1, \ldots, \operatorname{dim} N\right)$ are coordinates on $N$ and $\left(y^{a} ; a=1, \ldots, \operatorname{dim} M-\operatorname{dim} N\right)$ are coordinates for the typical fibre of $\pi$. It is not hard to see that the Euler-Lagrange operator for an intrinsically constrained Lagrangian system, when restricted to vertical directions becomes

$$
\mathcal{E} \mathcal{L}(L)^{v}=\frac{\partial L}{\partial y^{a}} d y^{a} .
$$


A critical curve $m(t)=\left(x^{i}(t), y^{a}(t)\right)$ of the Lagrangian system on $\pi: M \rightarrow N$ then satisfies the (possibly nonholonomic) condition

$$
\frac{\partial L}{\partial y^{a}}(x, \dot{x}, y)+F_{a}(x, y, \dot{x}, \dot{y})=0, \text { for } a=1, \ldots, \operatorname{dim} M-\operatorname{dim} N .
$$

The appearance of this constraint on the set of critical curves justifies the previous definitions. We now specialize to three distinct cases for constrained Lagrangian systems. We focus on a local treatment.

\subsection{Gyroscopic forces}

We study here the specific case where the non-conservative force term $F$ is of the form $F\left(v_{m}\right)=$ $-i_{v_{m}} \beta_{m}$ with $\beta$ a closed 2 -form on $M$. In this case we say that $F$ is a gyroscopic force term associated to $\beta$. As will become clear further on, Routh reduction of a Lagrangian system with non-abelian symmetry is in general a Lagrangian system subjected to gyroscopic forces. This type of forces therefore deserves special attention. Let $v_{m}=\left(x^{i}, y^{a}, \dot{x}^{i}, \dot{y}^{a}\right)$ in a coordinate system $\left(x^{i}, y^{a}\right)$, then $F$ assumes the following form

$$
F\left(v_{m}\right)=\left(\beta_{i j} \dot{x}^{j}+\beta_{i a} \dot{y}^{a}\right) d x^{i}+\left(\beta_{a b} \dot{y}^{b}-\beta_{i a} \dot{x}^{i}\right) d y^{a} .
$$

We say that the gyroscopic force term is regular if $\beta_{a b}$ is nondegenerate at all points in $M$, i.e. if $\beta$ is a nondegenerate 2 -form when restricted to $V \pi$. For gyroscopic forces, the intrinsic constraint (1) becomes:

$$
\frac{\partial L}{\partial y^{a}}+\left(\beta_{a b} \dot{y}^{b}-\beta_{i a} \dot{x}^{i}\right)=0
$$

and if $\beta$ is regular, it should be clear that this constraint can be brought into the form

$$
\dot{y}^{a}=-\left(\left(\left.\beta\right|_{V \pi}\right)^{-1}\right)^{a b}\left(\frac{\partial L}{\partial y^{b}}-\beta_{i b} \dot{x}^{i}\right) .
$$

An interesting property of intrinsically constrained Lagrangian systems with a gyroscopic force term is that they admit a presymplectic formulation on the manifold $T_{M} N$. To show this, we introduce the following maps:

1. the Legendre map $\mathbb{F}_{1} L: T_{M} N \rightarrow T_{M}^{*} N$, defined by

$$
\left\langle\mathbb{F}_{1} L\left(v_{n}, m\right),\left(w_{n}, m\right)\right\rangle=\left.\frac{d}{d \epsilon}\right|_{\epsilon=0} L\left(v_{n}+\epsilon w_{n}, m\right),
$$

for $\left(v_{n}, m\right),\left(w_{n}, m\right) \in T_{M} N$ arbitrary;

2. the energy $E_{L}$ of the Lagrangian $L$ is the function on $T_{M} N$ defined by

$$
E_{L}=\left\langle\mathbb{F}_{1} L\left(v_{n}, m\right),\left(v_{n}, m\right)\right\rangle-L\left(v_{n}, m\right)
$$

3. the projections $\pi_{1}: T_{M} N \rightarrow T N$ and $\pi_{2}: T_{M} N \rightarrow M$, and their analogues on the cotangent bundle: $\bar{\pi}_{1}: T_{M}^{*} N \rightarrow T^{*} N$ and $\bar{\pi}_{2}: T_{M}^{*} N \rightarrow M$.

We denote the Liouville form on $T^{*} N$ by $\theta_{N}$ and the associated symplectic 2 -form $\omega_{N}=d \theta_{N}$. The following result is then easily proven in a local coordinate system. 
Proposition 1 Let $m(t)$ denote a critical curve of the intrinsically constrained Lagrangian system $(M, L, F)$ with gyroscopic force term associated to $\beta$. Then $\gamma(t)=(\dot{n}(t), m(t)) \in T_{M} N$, with $n(t)=\pi(m(t))$, satisfies the following presymplectic equation

$$
\left.\left(i_{\dot{\gamma}(t)}\left(\left(\bar{\pi}_{1} \circ \mathbb{F}_{1} L\right)^{*} \omega_{N}+\pi_{2}^{*} \beta\right)=-d E_{L}\right)\right|_{\gamma}
$$

The converse also holds: any curve $\gamma(t) \in T_{M} N$ of the form $\gamma(t)=(\dot{n}(t), m(t))$ with $n(t)=$ $\pi(m(t))$ and that solves the above presymplectic equation, projects to a critical curve $m(t)=$ $\pi_{2}(\gamma(t))$ of $(M, L, F)$.

If (i) $\mathbb{F}_{1} L: T_{M} N \rightarrow T_{M}^{*} N$ is a diffeomorphism and if (ii) $\beta$ determines a regular gyroscopic force term, then the 2 -form $\left(\left(\bar{\pi}_{1} \circ \mathbb{F}_{1} L\right)^{*} \omega_{N}+\pi_{2}^{*} \beta\right)$ is symplectic. In [12] the symplectic structure of a Routh reduced system is proven to be of this type and it is identified with the the MarsdenWeinstein reduced symplectic structure of a Lagrangian system with symmetry. Without the above regularity assumptions (i) and (ii), the presymplectic system may only have solutions on a submanifold of $T_{M} N$. A next step would be to find these submanifolds, however, this is not the scope of this paper and we refer the reader to the presymplectic constraint algorithm [6, 7].

We end this paragraph with a slightly more general formulation of the above proposition. In general, the intrinsically constrained Lagrangian systems obtained from Routh reduction will have a force term $F=F_{1}+F_{2}$ that consists of two parts: a gyroscopic force term $F_{1}$ associated to $\beta$ and a force term $F_{2}=T^{*} \pi \circ \hat{F} \circ p_{1}$, with $\hat{F}: T_{M} N \rightarrow T^{*} N$. Again from local coordinate expressions, we conclude that critical curves of $(\pi: M \rightarrow N, L, F)$ solve the presymplectic equation (and vice versa)

$$
\left.\left(i_{\dot{\gamma}(t)}\left(\left(\bar{\pi}_{1} \circ \mathbb{F}_{1} L\right)^{*} \omega_{N}+\pi_{2}^{*} \beta\right)=-d E_{L}+\pi_{1}^{*} \hat{F}\right)\right|_{\gamma},
$$

with $\pi_{1}^{*} \hat{F}: T_{M} N \rightarrow T^{*}\left(T_{M} N\right)$ such that $\left\langle\pi_{1}^{*} \hat{F}\left(v_{n}, m\right), V_{\left(v_{n}, m\right)}\right\rangle=\left\langle\hat{F}\left(v_{n}, m\right), T \pi_{1}\left(V_{\left(v_{n}, m\right)}\right)\right\rangle$, with $\left(v_{n}, m\right) \in T_{M} N$ and $V_{\left(v_{n}, m\right)} \in T_{\left(v_{n}, m\right)}\left(T_{M} N\right)$ arbitrary.

\subsection{Regular configuration constraints}

Throughout this paragraph we assume that the force term $F$ is of the form $F=T^{*} \pi \circ \hat{F} \circ p_{1}$ with $\hat{F}: T_{M} N \rightarrow T^{*} N$ as above, that is, $F\left(v_{m}\right)=T_{m}^{*} \pi\left(\hat{F}\left(v_{n}, m\right)\right)$, with $v_{n}=T \pi\left(v_{m}\right)$. Then the constraint equation (1) reduces to

$$
\partial L / \partial y^{a}(x, \dot{x}, y)=0 .
$$

Intrinsic constraints of this form are called configuration constraints and they are said to be regular if a map $\gamma: T N \rightarrow M$, with $\pi \circ \gamma=\tau_{N}$ exists such that, in local coordinates, Eq. (2) is equivalent to $y^{a}=\gamma^{a}(x, \dot{x})$. As we show here, in this case critical curves of the Lagrangian system on $M$ are in a one to one correspondence to critical curves of a new Lagrangian system on $N$. The local existence of such a map $\gamma$ is guaranteed if the (vertical) Hessian of the Lagrangian is non-degenerate, i.e. if

$$
\operatorname{det} \frac{\partial^{2} L}{\partial y^{a} \partial y^{b}} \neq 0
$$

Consider the Lagrangian system $\left(N, L^{\prime}, F^{\prime}\right)$ on $N$, with

$$
L^{\prime}=L\left(v_{n}, \gamma\left(v_{n}\right)\right) \text { and }\left\langle F^{\prime}\left(v_{n}\right), w_{n}\right\rangle=\left\langle\hat{F}\left(v_{n}, \gamma\left(v_{n}\right)\right), w_{n}\right\rangle .
$$


Let $m(t)=\left(x^{i}(t), y^{a}(t)\right)$ be a curve in $M$. It is easily seen that the condition for $m$ to be critical w.r.t $(M \rightarrow N, L, F)$ is equivalent to $n(t)=\pi(m(t))$ being a critical curve of $\left(N, L^{\prime}, F^{\prime}\right)$. More precisely,

$$
\begin{aligned}
& \frac{d}{d t}\left(\frac{\partial L}{\partial \dot{x}^{i}}(x, \dot{x}, y)\right)=\frac{\partial L}{\partial x^{i}}(x, \dot{x}, y)+F_{i}(x, \dot{x}, y), \text { and } \\
& y^{a}=\gamma^{a}(x, \dot{x}) .
\end{aligned}
$$

is equivalent to

$$
\frac{d}{d t}\left(\frac{\partial L^{\prime}}{\partial \dot{x}^{i}}(x, \dot{x})\right)=\frac{\partial L^{\prime}}{\partial x^{i}}(x, \dot{x})+F_{i}(x, \gamma(x, \dot{x}), \dot{x})
$$

The correspondence is made explicit by the map $\gamma: \gamma(n(t))=m(t)$. In this sense the intrinsically constrained Lagrangian system $(M \rightarrow N, L, F)$ is 'equivalent' to the Lagrangian system $\left(N, L^{\prime}, F^{\prime}\right)$ on $N$.

The relation between Routh reduced systems and intrinsically constrained systems can now be made more explicit, and for this purpose we go ahead of some definitions and notations. We believe however that the reader familiar with geometric Routh reduction will benefit from the following. In general, a Routh reduced Lagrangian system will be an intrinsically constrained system on $M=\left(Q / G_{\mu} \times \tilde{\mathfrak{g}}\right)$ fibred over $N=Q / G$, where $Q$ is a manifold on on which a Lie group $G$ acts freely and properly. The bundle $M$ is a product bundle, and the Routhian is a Lagrangian on $T M$ independent of the velocities in the fibres of $M$. As will be made more precise in the following, this leads to constraints on the set of critical curves and due to the fact that $M$ is a product bundle we will distinguish two constraints. The constraints arising from the bundle $Q / G_{\mu} \rightarrow Q / G$ will always belong to the regular gyroscopic class. The constraints arising from the bundle $\tilde{\mathfrak{g}} \rightarrow Q / G$ will be configuration constraints. If these configuration constraints are regular, then we retrieve the standard Routh reduction procedure. The irregular case will sometimes lead to a variational problem on $N$ as is described in the next paragraph. In general, a Routh reduced system is associated to a presymplectic structure on $T_{M} N$, and its solutions can be constructed by means of the presymplectic constraint algorithm.

\subsection{Linear constraints and Lagrangian multipliers}

Throughout this paragraph we describe a special case of configuration constraints for a Lagrangian system on $M$ with the property that its critical curves correspond to the solutions of a variational problem on $N$. We assume that (i) $\pi: M \rightarrow N$ is a linear fibration and (ii) the force term $F$ is of the form $F=T^{*} \pi \circ \hat{F} \circ T \pi$, with $\hat{F}: T N \rightarrow T^{*} N$.

We say that the intrinsically constrained system $(M, L, F)$ is linear if $L$ is of the form

$$
L\left(v_{n}, m\right)=L_{0}\left(v_{n}\right)+\left\langle\alpha\left(v_{n}\right), m\right\rangle .
$$

with $\alpha: T N \rightarrow M^{*}$, fibred over the identity and $L_{0}$ a function on $T N$. The constraint equation (1D) for critical curves $m(t)=\left(x^{i}(t), y^{a}(t)\right)$ now becomes

$$
\frac{\partial L}{\partial y^{a}}(x, \dot{x}, y)=\alpha_{a}(x, \dot{x})=0 .
$$

Assume that 0 is a regular value of $\alpha$, and consider the submanifold $C$ of $T N$ defined by $v_{n} \in C$ if and only if $\alpha\left(v_{n}\right)=0$. The following proposition states that critical curves of $(\pi: M \rightarrow N, L, F)$ 
coincide with critical curves of the variational problem on $N$ with Lagrangian $L_{0}$ constrained to the submanifold $C$ in $T N$. Therefore, we can say that the initial Lagrangian system on $M$ admits a variational interpretation on the base manifold $N$, independent of the total space $M$. When working in a coordinate system, one might interpret the fibre coordinates $y^{a}$ as Lagrangian multipliers for the constrained variational problem on the base manifold.

Definition 4 A critical curve $p(t)$ of a Lagrangian system $\left(P, L^{\prime}, F^{\prime}\right)$ on a manifold $P$ constrained to a submanifold $C^{\prime}$ of $T P$ is a curve satisfying

$$
\delta \int_{I} L^{\prime}(\dot{p}(t)) d t+\int_{I}\left\langle F^{\prime}(\dot{p}(t)), \delta p(t)\right\rangle d t=0
$$

for any variation $\delta p$ with fixed endpoints originating from a deformation $p_{\epsilon}(t)$ of $p(t)$ which is entirely contained in $C^{\prime}$, i.e. $\dot{p}_{\epsilon}(t) \in C^{\prime}$.

Proposition 2 Given a linear intrinsically constrained Lagrangian system, $(\pi: M \rightarrow N, L=$ $\left.L_{0}+\langle\alpha, m\rangle, F\right)$ on $M$. Then the critical curves of $(\pi: M \rightarrow N, L, F)$ on $M$ project under $\pi$ onto critical curves of the Lagrangian system $\left(N, L_{0}, \hat{F}\right)$ on $N$ constrained to $C$.

Proof. The proposition is easily proven by noting that critical curves of $(\pi: M \rightarrow N, L, F)$ satisfy

$$
\delta \int_{I} L(\dot{m}(t)) d t+\int_{I}\langle\hat{F}(\dot{n}(t)), \delta n(t)\rangle d t=0
$$

for arbitrary variations with vanishing endpoints in $M$ and project onto curves in $C$. Among all these variations, we can now consider variations originating from deformations $m_{\epsilon}(t)$ of $m(t)$ that project onto deformations $n_{\epsilon}(t)$ of $n(t)$ that are contained in $C$. In that case the first term in the latter equation is precisely

$$
\left.\frac{d}{d \epsilon}\right|_{\epsilon} \int_{I} L\left(\dot{m}_{\epsilon}(t)\right) d t=\left.\frac{d}{d \epsilon}\right|_{\epsilon} \int_{I} L_{0}\left(\dot{n}_{\epsilon}(t)\right) d t
$$

and we thus find that $n(t)$ is a critical curve of $\left(N, L_{0}, \hat{F}\right)$ constrained to $C$.

\subsection{Connections}

We now return to the general setting of intrinsically constrained Lagrangian systems and conclude this section with a proposition which describes how to split up the Euler-Lagrange equations making use of a chosen connection on the fibration $\pi: M \rightarrow N$. A connection on $M$ is a direct sum decomposition of $T M$, i.e. a distribution $H$ on $M$ such that $T M=H \oplus V \pi$, and the associated map $h: T_{M} N \rightarrow H$ is called the horizontal lift. Using this connection we are now able to say that a variation $\delta m(t)$ of $m(t)$ is horizontal if $\delta m(t) \in H$, or if equivalently $\delta m(t)=h(m(t), \delta n(t))$, where $\delta n(t)$ is the corresponding variation of $n(t)=\pi(m(t))$. We denote the horizontal and vertical components of an arbitrary tangent vector $w$ by $w^{h}$ and $w^{v}$ respectively. The restriction of a cotangent vector $\alpha$ to the horizontal distribution is denoted by $\alpha^{h}$.

Proposition 3 The Euler-Lagrange operator of an arbitrary Lagrangian system $(M, L, F)$ on $M$ is completely determined if one only considers horizontal and vertical variations, i.e. if $m(t)$ is a critical curve of $(M, L, F)$ then it satisfies:

$$
\begin{aligned}
& \delta \int_{I} L(\dot{m}(t)) d t+\int_{I}\langle F(\dot{m}(t)), \delta m(t)\rangle d t=0 \text { with } \delta m^{v}(t)=0 \\
& \delta \int_{I} L(\dot{m}(t)) d t+\int_{I}\langle F(\dot{m}(t)), \delta m(t)\rangle d t=0 \text { with } \delta m^{h}(t)=0
\end{aligned}
$$


The first and second equations are equivalent to, respectively,

$$
\begin{aligned}
& \mathcal{E} \mathcal{L}(\ddot{m}(t))^{h}+F(\dot{m}(t))^{h}=0 \in T_{n}^{*} N \cong H_{m}, \\
& \mathcal{E} \mathcal{L}(\ddot{m}(t))^{v}+F(\dot{m}(t))^{v}=0 \in V_{m}^{*} \pi .
\end{aligned}
$$

Assume an adapted coordinate chart is chosen and that the horizontal distribution is spanned by the following set of vector fields

$$
\frac{\partial}{\partial x^{i}}-\Gamma_{i}^{a}(x, y) \frac{\partial}{\partial y^{a}},
$$

with $\Gamma_{i}^{a}$ the connection coefficients, then the horizontal and vertical parts of the Euler-Lagrange equations take the following form

$$
\begin{aligned}
& \frac{\partial L}{\partial x^{i}}-\frac{d}{d t}\left(\frac{\partial L}{\partial \dot{x}^{i}}\right)+F_{i}-\left(\frac{\partial L}{\partial y^{a}}+F_{a}\right) \Gamma_{i}^{a}=0, \\
& \frac{\partial L}{\partial y^{a}}+F_{a}=0 .
\end{aligned}
$$

In fact, by substituting the second in the first equation, we may conclude that these equations are independent of the connection coefficients.

\section{Lagrangian systems with symmetry}

We consider a Lagrangian system $(Q, L, F)$ on a manifold $Q$ and we assume that a Lie group $G$ freely and properly acts on $Q$. This ensures that $Q / G$ has a manifold structure and that $\pi: Q \rightarrow Q / G$ is a principal fibre bundle. The (right) action is denoted by $\Psi: G \times Q \rightarrow Q$ : $(g, q) \mapsto \Psi(q, g)=q g$. The associated maps $\sigma_{q}: G \rightarrow Q$ and $R_{g}: Q \rightarrow Q$ are defined by

$$
q g=\sigma_{q}(g)=R_{g}(q) .
$$

For the sake of simplicity, the infinitesimal action induced by $\sigma$ will be also denoted by $\sigma$ : $\sigma_{q}(\xi)=T \sigma_{q}(\xi)$, with $\xi \in \mathfrak{g}$ a Lie-algebra element of $G$. The vector field $\xi_{Q}$ on $Q$ is defined by $\xi_{Q}(q)=\sigma_{q}(\xi)$. We can consider the lifted action of $G$ on $T Q$, and using the previous definition on $T Q$, we shall write $\xi_{T Q}$ to denote the symmetry vector field associated with the Lie algebra element $\xi \in \mathfrak{g}$. Throughout this section we shall extensively rely on the choice of a principal connection on $Q$. This is a $\mathfrak{g}$-valued one-form $\omega$ on $Q$ satisfying $\omega\left(\xi_{Q}\right)=\xi$ and which is equivariant in the sense that, for arbitrary $g \in G$,

$$
R_{g}^{*} \omega=A d_{g^{-1}} \cdot \omega,
$$

where $A d$ denotes the adjoint action on $\mathfrak{g}$. We assume that the notions of horizontal lift, associated bundles and covariant derivatives, etc are well-known (see [10]). One typically defines the momentum map associated with the Lagrangian $L$ in the following way.

Definition 5 The momentum map $J_{L}: T Q \rightarrow \mathfrak{g}^{*}$ is the fibre derivative of $L$ restricted to vertical directions:

$$
J_{L}\left(v_{q}\right)(\xi)=\left.\frac{d}{d \epsilon}\right|_{\epsilon=0} L\left(v_{q}+\epsilon \xi_{Q}(q)\right) .
$$


It is well-known that if the Lagrangian is invariant under the (prolonged) action of $G$ on $T Q$, then (i) $J_{L}$ is an equivariant function, i.e. $R_{g}^{*} J_{L}=\mathrm{Ad}_{g}^{*} \cdot J_{L}$ and (ii) $J_{L}$ is a first integral of the critical curves of the conservative Lagrangian system $(Q, L, F=0)$.

Definition 6 A force term $F: T Q \rightarrow T^{*} Q$ is said to be invariant under the action of $G$ (or simply $G$-invariant) if the following two conditions are satisfied:

1. $\left\langle F\left(v_{q} g\right), w_{q} g\right\rangle=\left\langle F\left(v_{q}\right), w_{q}\right\rangle$

2. $\left\langle F\left(v_{q}\right), \xi_{Q}(q)\right\rangle=0$,

for $v_{q}, w_{q} \in T_{q} Q$ and $\xi \in \mathfrak{g}$ arbitrary.

A Lagrangian system $(Q, L, F)$ is $G$-invariant if the Lagrangian $L$ is an invariant function under the action of $G$ and if the force term $F$ is $G$-invariant.

A straightforward consequence of the definition of a $G$-invariant force term $F$ is that $F$ is reducible to a map $f$ between the quotient spaces $T Q / G$ and $T^{*}(Q / G)$. Moreover, the second condition in definition 6 guarantees that the momentum map $J_{L}$ is conserved along critical curves of a $G$-invariant Lagrangian system $(Q, L, F)$ (it is sufficient, but not necessary). In the following proposition we study the necessary conditions for a Lagrangian system $(Q, L, F)$ such that $J_{L}$ is conserved along its critical curves. This generality will be of importance further on when considering the Routhian and its momentum map, both associated with an invariant Lagrangian.

Proposition 4 The momentum map for a (not necessarily G-invariant) Lagrangian system $(Q, L, F)$ is conserved if and only if the force term is such that $\left\langle d L, \xi_{T Q}\right\rangle=-\left\langle F, \xi_{Q}\right\rangle$ for all $\xi \in \mathfrak{g}$ along the critical curves. If this holds we say that the Lagrangian system $(Q, L, F)$ has conserved momentum $J_{L}$ w.r.t. the action of $G$.

Proof. Assume that $q(t)$ is a critical curve of the Lagrangian system $(L, F)$. We now compute the derivative of the momentum map along the trajectory and proof that it is equal to zero. Let $\left(q^{i}\right)$ be a local coordinate system on $Q$ and let $\xi \in \mathfrak{g}$ be chosen arbitrarily, then

$$
\begin{aligned}
\left.\frac{d}{d t}\right|_{t}\left(J_{L}(\dot{q}(t))(\xi)\right) & =\left.\left.\frac{d}{d t}\right|_{t} \frac{d}{d \epsilon}\right|_{\epsilon=0} L\left(\dot{q}(t)+\epsilon \xi_{Q}(q(t))=\left.\frac{d}{d t}\right|_{t}\left(\frac{\partial L}{\partial \dot{q}^{i}} \xi_{Q}^{i}\right)\right. \\
& =\frac{d}{d t}\left(\frac{\partial L}{\partial \dot{q}^{i}}\right) \xi_{Q}^{i}+\frac{\partial L}{\partial \dot{q}^{i}} \frac{\partial \xi_{Q}^{i}}{\partial q^{j}} \dot{q}^{j} \\
& =\frac{\partial L}{\partial q^{i}} \xi_{Q}^{i}+F_{i} \xi_{Q}^{i}+\frac{\partial L}{\partial \dot{q}^{i}} \frac{\partial \xi_{Q}^{i}}{\partial q^{j}} \dot{q}^{j} \\
& =\xi_{T Q}(L)(\dot{q}(t))+\left\langle F(\dot{q}(t)), \xi_{Q}(q(t))\right\rangle=0 .
\end{aligned}
$$

Note that, if $L$ is invariant then the momentum map is conserved along critical curves if $F$ satisfies condition (2) from definition 6 .

The first step in our approach to Routh reduction of a $G$-invariant Lagrangian system $(Q, L, F)$ consists of defining an equivalent Lagrangian system on $Q$ whose Lagrangian is only invariant under the the action of a subgroup of $G$ although it has conserved momentum w.r.t. the action of $G$. This observation should justify the above definitions. 


\subsection{General constructions on the quotient spaces}

Assume throughout the remaining of this paper that a regular value $\mu \in \mathfrak{g}^{*}$ for the momentum map of a $G$-invariant Lagrangian system $(Q, L, F)$ is chosen. We denote the group $G_{\mu}$ as the isotropy subgroup of $G$ of $\mu$ under the coadjoint action of $G$ on $\mathfrak{g}^{*}$.

This section describes the structure of the manifold $T Q / G_{\mu}$ on which the Routhian lives and how variations of a curve in $Q$ behave when projected onto the quotient $T Q / G_{\mu}$. Many of the properties explained below can be found in more detail in [2]. We start with fixing notations and defining several projections and bundles appearing in the chosen framework. Recall that the bundle $Q / G_{\mu} \rightarrow Q / G$ is an associated bundle of the principal fibre bundle $Q \rightarrow Q / G$, whose typical fibre is the orbit $\mathcal{O}_{\mu}$ of $\mu$ under the adjoint action on $\mathfrak{g}^{*}$.

Definition 7 The projections onto the different quotient spaces are denoted by

$$
\begin{aligned}
\pi: Q & \rightarrow Q / G, & & \pi_{\mu}: Q \rightarrow Q / G_{\mu}, \\
\bar{\pi}_{\mu}: Q / G_{\mu} & \rightarrow Q / G, & & \tilde{\pi}: \tilde{\mathfrak{g}}:=(Q \times \mathfrak{g}) / G \rightarrow Q / G .
\end{aligned}
$$

A point in $Q, Q / G_{\mu}$ and $Q / G$ is typically denoted by $q, y$ and $x$ respectively. Similarly, tangent vectors in $T_{q} Q, T_{y}\left(Q / G_{\mu}\right)$ and $T_{x}(Q / G)$ are denoted by $v_{q}, v_{y}$ and $v_{x}$ respectively. Further, elements in the bundle $\tilde{\mathfrak{g}} \rightarrow Q / G$ associated to the Lie-algebra $\mathfrak{g}$ are denoted by $\tilde{\xi}=[q, \xi]_{G}$, with $\xi \in \mathfrak{g}, q \in Q$.

If a principal connection $\omega$ on $\pi: Q \rightarrow Q / G$ is chosen, then the horizontal lift of a tangent vector in $T(Q / G)$ to the point $q \in \pi^{-1}(x)$ is denoted by $\left(v_{x}\right)_{q}^{h}$. The horizontal lift of a curve $x(t)$ from $Q / G$ to $Q$ is denoted by $q_{h}(t)$

According to our previous notation conventions, an element in the product bundle $T(Q / G) \times$ $Q / G_{\mu} \times \tilde{\mathfrak{g}} \rightarrow Q / G$ is denoted by $\left(v_{x}, y, \tilde{\xi}\right)$, where $v_{x} \in T_{x}(Q / G), y \in Q / G_{\mu}$ and $\tilde{\xi} \in \tilde{\mathfrak{g}}$ satisfy $\bar{\pi}_{\mu}(y)=\tilde{\pi}(\tilde{\xi})=x \in Q / G$.

Proposition 5 The manifold $T Q / G_{\mu}$ is isomorphic to $T(Q / G) \times Q / G_{\mu} \times \tilde{\mathfrak{g}}$. The isomorphism can be made explicit with the choice of a principal connection $\omega$ on $Q$.

Proof. Assume that a connection $\omega$ on $Q$ is chosen. Let $\left[v_{q}\right]_{G_{\mu}} \in T Q / G_{\mu}$ be the orbit of an arbitrary tangent vector $v_{q}$ in $T Q$. We now define a map

$$
\phi_{\omega}: T Q / G_{\mu} \rightarrow T(Q / G) \times Q / G_{\mu} \times \tilde{\mathfrak{g}}
$$

together with its inverse $\psi_{\omega}$ as

$$
\begin{aligned}
& \phi_{\omega}\left(\left[v_{q}\right]_{G_{\mu}}\right)=\left(T \pi\left(v_{q}\right), \pi_{\mu}(q),\left[q, \omega(q)\left(v_{q}\right)\right]_{G}\right) \\
& \psi_{\omega}\left(v_{x}, y, \tilde{\xi}\right)=\left[\left(v_{x}\right)_{q}^{h}+\sigma_{q}(\xi)\right]_{G_{\mu}}, \text { with } y=[q]_{G_{\mu}} \text { and } \tilde{\xi}=[q, \xi]_{G} .
\end{aligned}
$$

It is standard to show that these maps are smooth and do not depend on the choice of an element in the orbits under consideration.

Using similar arguments as before, one can show that $T Q / G \cong T(Q / G) \times \tilde{\mathfrak{g}}$. Taking into account these isomorphisms, the natural projection $\lambda_{\mu}: T Q / G_{\mu} \rightarrow T Q / G$ simply reads

$$
\lambda_{\mu}\left(v_{x}, y, \tilde{\xi}\right)=\left(v_{x}, \tilde{\xi}\right)
$$


Definition 8 An invariant Lagrangian function $L$ on $T Q$ reduces to a function $l$ on $T Q / G$, defined by

$$
l\left(v_{x}, \tilde{\xi}\right)=L\left(\left(v_{x}\right)_{q}^{h}+\sigma_{q}(\xi)\right), \text { with } q \in \pi^{-1}(x),[q, \xi]_{G}=\tilde{\xi} .
$$

The function $\lambda_{\mu}^{*} l$, defined on $T Q / G_{\mu}$ is denoted by the same symbol.

The associated momentum map $J_{L}: T Q \rightarrow \mathfrak{g}^{*}$, reduces to a bundle map $j_{l}: T Q / G \rightarrow \tilde{\mathfrak{g}}^{*}$, fibred over the identity on $Q / G$ and defined by

$$
\left\langle j_{l}\left(v_{x}, \tilde{\xi}\right), \tilde{\eta}\right\rangle=\left\langle J_{L}\left(\left(v_{x}\right)_{q}^{h}+\sigma_{q}(\xi)\right), \eta\right\rangle \text {, with } q \in \pi^{-1}(x),[q, \xi]_{G}=\tilde{\xi},[q, \eta]_{G}=\tilde{\eta} .
$$

The reduced map $j_{l}$ can alternatively be defined from $l$ directly. Let $\mathbb{F}_{\tilde{\xi}} l: T(Q / G) \times \tilde{\mathfrak{g}} \rightarrow T(Q / G) \times$ $\tilde{\mathfrak{g}}^{*}$ be the fiber derivative of $l$ with respect to the (linear) factor $\tilde{\mathfrak{g}}$.

Proposition 6 The maps $j_{l}$ and $l$ are related by:

$$
\left\langle\mathbb{F}_{\tilde{\xi}} l\left(v_{x}, \tilde{\xi}\right),\left(v_{x}, \tilde{\eta}\right)\right\rangle=\left\langle j_{l}\left(v_{x}, \tilde{\xi}\right), \tilde{\eta}\right\rangle .
$$

Proof. Let $\left(v_{x}, \tilde{\xi}\right),\left(v_{x}, \tilde{\eta}\right) \in T(Q / G) \times \tilde{\mathfrak{g}}$ be arbitrary. Fix a point $q \in Q$ projecting to $x$ such that $[q, \xi]_{G}=\tilde{\xi}$ and $[q, \eta]_{G}=\tilde{\eta}$; then

$$
\begin{aligned}
\left\langle\mathbb{F}_{\tilde{\xi}} l\left(v_{x}, \tilde{\xi}\right),\left(v_{x}, \tilde{\eta}\right)\right\rangle & :=\left.\frac{d}{d \epsilon}\right|_{\epsilon=0} l\left(v_{x}, \tilde{\xi}+\epsilon \tilde{\eta}\right)=\left.\frac{d}{d \epsilon}\right|_{\epsilon=0} L\left(\left(v_{x}\right)_{q}^{h}+\sigma_{q}(\xi+\epsilon \eta)\right) \\
& =\left\langle J_{L}\left(\left(v_{x}\right)_{q}^{h}+\sigma_{q}(\xi)\right), \eta\right\rangle=\left\langle j_{l}\left(v_{x}, \tilde{\xi}\right), \tilde{\eta}\right\rangle .
\end{aligned}
$$

The element $\mu \in \mathfrak{g}^{*}$ defines a map $\tilde{\mu}: Q / G_{\mu} \rightarrow \tilde{\mathfrak{g}}^{*}$ such that

$$
\langle\tilde{\mu}(y), \tilde{\xi}\rangle=\langle\mu, \xi\rangle \text {, with }[q]_{G_{\mu}}=y \text { and }[q, \xi]_{G}=\tilde{\xi} \text {. }
$$

Due to the equivariance of $J_{L}$, the level set $J_{L}^{-1}(\mu)$ is $G_{\mu}$ invariant and reduces to a subset of $T Q / G_{\mu}$.

Proposition 7 The quotient manifold $J_{L}^{-1}(\mu) / G_{\mu}$ is identified with the subset of points $\left(v_{x}, y, \tilde{\xi}\right)$ in $T Q / G_{\mu}$ satisfying $j_{l}\left(v_{x}, \tilde{\xi}\right)=\tilde{\mu}(y)$.

In classical Routhian reduction, an essential condition for reducibility of an invariant Lagrangian system is that this submanifold $J_{L}^{-1}(\mu) / G_{\mu}$ is precisely $T(Q / G) \times Q / G_{\mu}$. This situation is the subject of the next session.

\subsection{The regular case}

Definition 9 An invariant Lagrangian $L$ is said to be $G$-regular if the map $\mathbb{F}_{\tilde{\xi}}$ lis a diffeomorphism between $T(Q / G) \times \tilde{\mathfrak{g}}$ and $T(Q / G) \times \tilde{\mathfrak{g}}^{*}$.

We shall write the inverse of this map as $\kappa_{l}: T(Q / G) \times \tilde{\mathfrak{g}}^{*} \rightarrow T(Q / G) \times \tilde{\mathfrak{g}}$.

Proposition 8 If $L$ is $G$-invariant and $G$-regular then the mapping

$$
\begin{aligned}
J_{L}^{-1}(\mu) / G_{\mu} & \rightarrow T(Q / G) \times Q / G_{\mu} \\
{\left[v_{q}\right]_{G_{\mu}} } & \mapsto\left(T \pi\left(v_{q}\right),[q]_{G_{\mu}}\right)
\end{aligned}
$$

is a diffeomorphism. 
Proof. We provide the inverse of the mapping of the statement. Given a point $\left(v_{x}, y\right) \in$ $T(Q / G) \times Q / G_{\mu}$, and let $\tilde{\xi} \in \tilde{\mathfrak{g}}$ be such that

$$
\left(v_{x}, \tilde{\xi}\right)=\kappa_{l}\left(v_{x}, \tilde{\mu}(y)\right)
$$

Fix a representant $q$ for $y$, i.e. $y=[q]_{G_{\mu}}$, and let $\xi$ be such that $\tilde{\xi}=[q, \xi]_{G}$. Then the point $v_{q}=\left(\left(v_{x}\right)_{q}^{h}+\xi_{Q}(q)\right) \in T Q$ belongs to the set $J_{L}^{-1}(\mu)$. Indeed, with $\tilde{\eta}=[q, \eta]_{G}$ arbitrary,

$$
J_{L}\left(\left(v_{x}\right)_{q}^{h}+\sigma_{q}(\xi)\right)(\eta)=\left\langle j_{l}\left(v_{x}, \tilde{\xi}\right), \tilde{\eta}\right\rangle=\langle\tilde{\mu}(y), \tilde{\eta}\rangle=\langle\mu, \eta\rangle .
$$

It only remains to show that this definition is independent of the chosen representative $q$ at $y$. Due to the equivariance of $J_{L}$ this is standard. Finally, with $\left(v_{x}, y\right)$ we can now define an element in $J_{L}^{-1}(\mu) / G_{\mu}$ determined as the orbit of $v_{q}$. The mapping from $T(Q / G) \times Q / G_{\mu}$ to $J_{L}^{-1}(\mu)$ obtained in this way is the inverse of the map defined in the statement of the proposition.

Remark 1 Note that the mapping given in Proposition 8 is precisely the first two components of the diffeomorphism (3) in Proposition 5. In other words, the G-regularity shows that the submanifold $J_{L}^{-1}(\mu) / G_{\mu}$ in $T(Q / G) \times Q / G_{\mu} \times \tilde{\mathfrak{g}}$ can be given as the set of points $\left(v_{x}, y, \tilde{\xi}\right)$ with $\left(v_{x}, y\right) \in T(Q / G) \times Q / G_{\mu}$ arbitrary and with $\tilde{\xi}$ such that $\left(v_{x}, \tilde{\xi}\right)=\kappa_{l}\left(v_{x}, \tilde{\mu}(y)\right)$.

With this in mind, it follows that the converse of Proposition 8 is also valid: if for any $\mu \in \mathfrak{g}^{*}$ the map $J_{L}^{-1}(\mu) / G_{\mu} \rightarrow T(Q / G) \times Q / G_{\mu}$ is a diffeomorphism, then $L$ is $G$-regular. This shows that our definition of $G$-regular Lagrangians is independent of the connection $\omega$.

Remark 2 Let $\rho$ be a $G$-invariant Riemannian metric on $Q$ and $V \in C^{\infty}(Q)$ a $G$-invariant potential energy. The Lagrangian $L\left(v_{q}\right)=\frac{1}{2} \rho\left(v_{q}, v_{q}\right)-V(q)$ (kinetic minus potential) is $G$-regular (see, for example, [15]).

Note however that a Lagrangian can be both hyperregular (i.e., its Legendre transformation is a diffeomorphism) and $G$-invariant but not $G$-regular. Recall the example from the introduction $L=\left(\dot{q}^{1}\right)^{2}+\dot{q}^{1} \dot{q}^{2}-V\left(q^{1}\right)$ with $V \in C^{\infty}(\mathbb{R}), Q=\mathbb{R}^{2}$ and $G=\mathbb{R}$ acting by translations in the $q^{2}$ coordinate. Fix a connection, say $\omega=d q^{2}$, and let $\tilde{\xi}=\left(q^{1}, \xi\right), \tilde{\eta}=\left(q^{1}, \eta\right)$ be arbitrary in $\tilde{\mathfrak{g}}=Q / G \times \mathbb{R}$. Then the reduced Lagrangian $l: T(Q / G) \times \tilde{\mathfrak{g}} \rightarrow \mathbb{R}$ is $l\left(q^{1}, \dot{q}^{1}, \xi\right)=\left(\dot{q}^{1}\right)^{2}+\dot{q}^{1} \xi-V\left(q^{1}\right)$ and $\left\langle\mathbb{F} l_{\tilde{\xi}}\left(q^{1}, \dot{q}^{1}, \xi\right),\left(q^{1}, \dot{q}^{1}, \eta\right)\right\rangle=\dot{q}^{1} \eta$. Thus $\mathbb{F} l_{\tilde{\xi}}$ is not a diffeomorphism.

It is standard in Routh reduction to introduce the following definition, although due to the fact that $L$ may not be of type 'kinetic minus potential' the definition is slightly more general than the usual definition of the locked inertia tensor.

Definition 10 Given a Lagrangian $L: T Q \rightarrow \mathbb{R}$ and a point $v_{q} \in T Q$, the mapping $\mathcal{I}_{v_{q}}: \mathfrak{g} \rightarrow \mathfrak{g}^{*}$ defined by

$$
\begin{aligned}
\mathcal{I}_{v_{q}}(\xi)(\eta) & =\left.\left.\frac{d}{d \epsilon}\right|_{\epsilon=0} \frac{d}{d \tau}\right|_{\tau=0} L\left(v_{q}+\epsilon \xi_{Q}(q)+\tau \eta_{Q}(q)\right) \\
& =\left.\frac{d}{d \tau}\right|_{\tau=0} J_{L}\left(v_{q}+\tau \eta_{Q}(q)\right)(\xi),
\end{aligned}
$$

for $\xi, \eta \in \mathfrak{g}$ arbitrary, is called the locked intertia tensor at $v_{q}$.

We say that $L$ is locally $G$-regular if the locked-inertial tensor $\mathcal{I}_{v_{q}}$ is invertible for any $v_{q} \in T Q$. Local $G$-regular Lagrangians only guarantee that $J_{L}^{-1}(\mu)$ is locally diffeomorphic to $T(Q / G) \times$ $Q / G_{\mu}$. In any case, local $G$-regularity presents the advantage of being a property easy to check (besides the applications of the locked inertia tensor in other situations as stability, cf. [13]). We refer to [4 for a description of Routh reduction for locally $G$-regular Lagrangians. 


\subsection{The reduced form induced by the connection}

We now return to the general case and continue defining additional objects living on the quotient spaces under consideration. These objects will appear as force terms when studying Routhian reduced systems. Recall that we fixed a principal connection $\omega$ on $Q$. The one-form on $Q$ obtained by pairing $\mu$ with the $\mathfrak{g}$-valued one-form $\omega$ is denoted by $\omega^{\mu}=\langle\mu, \omega\rangle$. The Lie-algebra $\mathfrak{g}_{\mu}$ of the isotropy subgroup $G_{\mu}$ is a subalgebra of $\mathfrak{g}$ which can alternatively be determined as the set of elements $\xi \in \mathfrak{g}$ satisfying $\operatorname{ad}_{\xi}^{*} \mu=0$.

Proposition 9 The two-form $d \omega^{\mu}=\langle\mu, d \omega\rangle$ on $Q$ is projectable to a two-form on $Q / G_{\mu}$, denoted by $\beta^{\mu}$.

The invariant force form $F: T Q \rightarrow T^{*} Q$ reduces to a map: $f: T Q / G \rightarrow T^{*}(Q / G)$.

Proof. It is easily seen that $d \omega^{\mu}$ is invariant under $G_{\mu}$ and annihilates vertical tangent vectors in $Q \rightarrow Q / G_{\mu}$. The latter follows from the equivariance of $\omega$ : let $\xi$ be an arbitrary element in $\mathfrak{g}_{\mu}$, then

$$
\begin{aligned}
i_{\xi_{Q}} d \omega^{\mu} & =\left\langle\mu, \mathcal{L}_{\xi_{Q}} \omega\right\rangle-\left\langle\mu, d i_{\xi_{Q}} \omega\right\rangle \\
& =\left\langle\mu,-\operatorname{ad}_{\xi} \omega\right\rangle=-\left\langle\operatorname{ad}_{\xi}^{*} \mu, \omega\right\rangle=0
\end{aligned}
$$

The second part of the proposition is a straightforward consequence of Definition 6 of an invariant force term. Next we study the bundle $\bar{\pi}_{\mu}: Q / G_{\mu} \rightarrow Q / G$ and in particular the set of tangent vectors vertical to $\bar{\pi}_{\mu}$.

First note that both $\pi: Q \rightarrow Q / G$ and $\pi_{\mu}: Q \rightarrow Q / G_{\mu}$ are principal fibres bundles (from now on PFB), with structure group $G$ and $G_{\mu}$ respectively. The adjoint bundle $\tilde{\mathfrak{g}}$ is the bundle associated to $\mathfrak{g}$ w.r.t the PFB $\pi$. Similarly we can consider the adjoint bundle $\tilde{\mathfrak{g}}_{\mu}$ associated to $\mathfrak{g}_{\mu}$ w.r.t. the PFB $\pi_{\mu}$. It is not hard to see that $\tilde{\mathfrak{g}}_{\mu}$ is a subbundle of the fibred product $Q / G_{\mu} \times \tilde{\mathfrak{g}}$ : let $[q, \xi]_{G_{\mu}} \in \tilde{\mathfrak{g}}_{\mu}$ be arbitrary, then we define an element in $Q / G_{\mu} \times \tilde{\mathfrak{g}}$ by fixing a representative $(q, \xi) \in Q \times \mathfrak{g}_{\mu}$ and we let $\left(y=\pi_{\mu}(q)=y, \tilde{\xi}=[q, \xi]_{G}\right)$ determine the corresponding element in $Q / G_{\mu} \times \tilde{\mathfrak{g}}$. The Lie algebra bracket $[\cdot, \cdot]$ on $\mathfrak{g}$ carries over to a Lie algebra structure on the fibres of $\tilde{\mathfrak{g}}$. The bracket is denoted with the same symbol: $[\tilde{\xi}, \tilde{\eta}]=[q,[\xi, \eta]]_{G}$, with $[q, \xi]_{G}=\tilde{\xi}$ and $[q, \eta]_{G}=\tilde{\eta}$ arbitrary. For notational convenience we introduce the following shorthand notations: $\bar{\pi}_{\mu}^{*} \tilde{\mathfrak{g}}=Q / G_{\mu} \times \tilde{\mathfrak{g}}$, and the dual bundle by $\bar{\pi}_{\mu}^{*} \tilde{\mathfrak{g}}^{*}=Q / G_{\mu} \times \tilde{\mathfrak{g}}^{*}$. Since $\tilde{\mathfrak{g}}_{\mu}$ is a subbundle of $\bar{\pi}_{\mu}^{*} \tilde{\mathfrak{g}}$ we may consider the quotient bundle: $\bar{\pi}_{\mu}^{*} \tilde{\mathfrak{g}} / \tilde{\mathfrak{g}}_{\mu}$. Finally, note that $\tilde{\mu}: Q / G_{\mu} \rightarrow \tilde{\mathfrak{g}}^{*}$ can be seen as section of $Q / G_{\mu} \times \tilde{\mathfrak{g}}^{*} \rightarrow Q / G_{\mu}$.

Definition 11 Associated with the map $\tilde{\mu}: Q / G_{\mu} \rightarrow \tilde{\mathfrak{g}}^{*}$ we define a section $\operatorname{ad}^{*} \tilde{\mu}$ of $\bigwedge^{2} \bar{\pi}_{\mu}^{*} \tilde{\mathfrak{g}}^{*} \rightarrow$ $Q / G_{\mu}$ in the following way

$$
\operatorname{ad}^{*} \tilde{\mu}(y)((y, \tilde{\xi}),(y, \tilde{\eta}))=\langle\tilde{\mu}(y),[\tilde{\xi}, \tilde{\eta}]\rangle
$$

where $\tilde{\xi}, \tilde{\eta} \in \tilde{\mathfrak{g}}_{x}, \pi_{\mu}(y)=x$ arbitrary (recall that $\left.\bar{\pi}_{\mu}^{*} \tilde{\mathfrak{g}}=Q / G_{\mu} \times \tilde{\mathfrak{g}}\right)$.

We will use the following shorthand notations: $\operatorname{ad}^{*} \tilde{\mu}(y)(\tilde{\xi}, \tilde{\eta})$ or $\operatorname{ad}_{\tilde{\xi}}^{*} \tilde{\mu}(y)=i_{\tilde{\xi}}\left(\operatorname{ad}^{*} \tilde{\mu}(y)\right)$. The twoform $\operatorname{ad}^{*} \tilde{\mu}$ is clearly antisymmetric and its kernel consists precisely of elements in $\tilde{\mathfrak{g}}_{\mu}$. Therefore, without introducing a new symbol, we can regard $\operatorname{ad}^{*} \tilde{\mu}$ as a section of $\bigwedge^{2}\left(\bar{\pi}_{\mu}^{*} \tilde{\mathfrak{g}} / \tilde{\mathfrak{g}}_{\mu}\right)^{*} \rightarrow Q / G_{\mu}$ which is nondegenerate as a map $\bar{\pi}_{\mu}^{*} \tilde{\mathfrak{g}} / \tilde{\mathfrak{g}}_{\mu} \rightarrow\left(\bar{\pi}_{\mu}^{*} \tilde{\mathfrak{g}} / \tilde{\mathfrak{g}}_{\mu}\right)^{*}$. Recall that by choosing a point $q \in$ $\pi_{\mu}^{-1}(y)$, the fibre of the subbundle $\tilde{\mathfrak{g}}_{\mu}$ at the point $y$ is isomorphic to $\mathfrak{g}_{\mu}$, the Lie algebra of the isotropy subgroup $G_{\mu}$. This observation brings us to the next proposition. 
Proposition 10 The bundle $V \bar{\pi}_{\mu} \rightarrow Q / G_{\mu}$ of vertical tangent vectors to the fibration $\bar{\pi}_{\mu}$ : $Q / G_{\mu} \rightarrow Q / G$ is isomorphic to $\bar{\pi}_{\mu}^{*} \tilde{\mathfrak{g}} / \tilde{\mathfrak{g}}_{\mu} \rightarrow Q / G_{\mu}$.

Proof. We define a surjective linear map from $\bar{\pi}_{\mu}^{*} \tilde{\mathfrak{g}}$ to $V \bar{\pi}_{\mu}$, in the following way. Fix a point $(y, \tilde{\xi}) \in \bar{\pi}_{\mu}^{*} \tilde{\mathfrak{g}}$ such that $y=[q]_{G_{\mu}}$ and $\bar{\pi}_{\mu}(y)=x$. Let $\xi$ be a representant of $\tilde{\xi}$ at the point $q$, i.e. $\tilde{\xi}=[q, \xi]_{G}$, and consider the vertical tangent vector in $V_{y} \bar{\pi}_{\mu}$ obtained by $T \pi_{\mu}\left(\sigma_{q}(\xi)\right)$. It is clear that this map is well defined (i.e. independent of the choice of the point $q$ in $[q]_{G_{\mu}}$ and that it is onto. Furthermore, the kernel of this map consists of the elements in $\tilde{\mathfrak{g}}_{\mu}$ at the point $y$. For later purposes it is important to see that the tangent vectors to the vertical curves $[q g(t)]_{G_{\mu}} \in \bar{\pi}_{\mu}^{-1}(x)$ through $y$ with $g(0)=e$ and $\xi=\dot{g}(0) \in \mathfrak{g}$ are mapped onto $\left(y,[q, \xi]_{G}\right)+\tilde{\mathfrak{g}}_{\mu}$ at the point $y$. We conclude with recalling the definition of a connection on an arbitrary associated bundle corresponding to the fixed principal connection $\omega$ on $Q$. Let $E$ denote a manifold on which $G$ acts from the left.

Definition 12 The connection or horizontal distribution on the associated bundle $Q \times{ }_{G} E$ corresponding with the principal connection $\omega$ is defined as the set of tangent vectors to curves of the form $[q(t), f]_{G}$ with $q(t) \in Q$ horizontal and $f \in E$ arbitrary. In the case that $F$ is a linear space on which $G$ acts linearly, we define the covariant derivative $D$ as

$$
\frac{D}{D t}[q(t), e(t)]_{G}=\left[q(t),\left(\omega_{q(t)}(\dot{q}(t))\right) \cdot e(t)\right]_{G}+[q(t), \dot{e}(t)]_{G} .
$$

It is understood that in the above definition the action of a Lie-algebra element $\omega_{q}(\dot{q})$ on the element $e \in E$ is denote by $\cdot$

We will use the connection in associated bundles in the following two cases:

Firstly, the principal connection $\omega$ determines a connection in $Q / G_{\mu} \rightarrow Q / G$, the bundle associated to the orbit space $\mathcal{O}_{\mu}$. The tangent space to $Q / G_{\mu}$ can be written as a direct sum of the horizontal and vertical distribution:

$$
T\left(Q / G_{\mu}\right) \cong \bar{\pi}_{\mu}^{*} T(Q / G) \oplus_{Q / G_{\mu}} \bar{\pi}_{\mu}^{*} \tilde{\mathfrak{g}} / \tilde{\mathfrak{g}}_{\mu} .
$$

This in turn implies that the two-form $\beta^{\mu}$ (cf. Proposition 9 above) can be decomposed into a horizontal-horizontal and vertical-vertical part (the horizontal-vertical part vanishes)

$$
\beta^{\mu}=\left(\tilde{\Omega}^{\mu},-\operatorname{ad}^{*} \tilde{\mu}\right),
$$

where

$$
\tilde{\Omega}^{\mu} \quad: \quad Q / G_{\mu} \rightarrow \wedge^{2} T^{*}(Q / G) ; y \mapsto\langle\tilde{\mu}(y), \tilde{\Omega}\rangle
$$

$\tilde{\Omega}$ being the $\tilde{\mathfrak{g}}$-valued curvature 2 -form on $Q / G$. Such a decomposition is a straightforward consequence of the structure equation $\Omega=d \omega+[\omega, \omega]$ and the fact that in this specific case the horizontal distribution on $Q / G_{\mu}$ is the projection of the horizontal distribution on $Q$.

Secondly, if $E=\mathfrak{g}$ the covariant derivative on the adjoint bundle $\tilde{\mathfrak{g}}$ equals, for any $q(t) \in Q$ and $\xi(t) \in \mathfrak{g}$ (with $[\cdot, \cdot]$ the Lie bracket on $\mathfrak{g}$ ):

$$
\frac{D}{D t}[q(t), \xi(t)]_{G}=\left[q(t),\left[\omega_{q(t)}(\dot{q}(t)), \xi(t)\right]\right]_{G}+[q(t), \dot{\xi}(t)]_{G} .
$$




\subsection{Variations}

The notions introduced here are described in more detail in 2. For the sake of completeness and because we work in a slightly different situation we nevertheless briefly introduce the notions of vertical and horizontal variations in the context of Lagrangian systems with symmetry. The main point is to consider a variation of a curve $q(t)$ in the total space $Q$, and subsequently study the projection of this variation to the quotient spaces $Q / G_{\mu}$ and $Q / G$.

For that purpose, we fix a curve in $q(t)$ in $Q$ and its lift $\dot{q}(t)$ in $T Q$. Following the notations from the previous section, we shall write the projection of $\dot{q}(t)$ to $T Q / G_{\mu}$ as $(\dot{x}(t), y(t), \tilde{\xi}(t))$, where $\dot{x}(t)=T_{q(t)} \pi(\dot{q}(t)), y(t)=\pi_{\mu}(q(t))$ and $\tilde{\xi}(t)=\left[q(t), \omega_{q(t)}(\dot{q}(t))\right]_{G}$.

Definition $13 A$ deformation of the curve $q(t)$ is smooth function $q(t, \epsilon)=q_{\epsilon}(t)$ such that $q(t, 0)=q(t)$. The corresponding variation is defined by

$$
\delta q(t)=\left.\frac{\partial q(t, \epsilon)}{\partial \epsilon}\right|_{\epsilon=0} \in T_{q(t)} Q
$$

1. A vertical deformation is a deformation that can be written as $q(t, \epsilon)=q(t) g_{\epsilon}(t)$, where the family of curves $g_{\epsilon}(t) \in G$ satisfies $g_{0}(t)=e$, with e the unity of $G$.

2. A variation $\delta q(t)$ such that $T \pi_{q(t)}(\delta q(t))=0$ for all $t$ is said to be a vertical variation.

3. A variation $\delta q(t)$ such that $\omega(q(t))(\delta q(t))=0$ for all $t$ is said to be a horizontal variation.

Obviously, the variation induced by a vertical deformation is vertical and it is not hard to see that any vertical variation can be given in that way.

Given an arbitrary vertical deformation $q(t, \epsilon)=q(t) g_{\epsilon}(t)$, then the projection of $(q(t, \epsilon), \dot{q}(t, \epsilon))$ to the quotient space $T Q / G_{\mu}$ is written as $(\dot{x}(t), y(t, \epsilon), \tilde{\xi}(t, \epsilon))$, where according to the previous definitions:

$$
\begin{aligned}
y(t, \epsilon) & =[q(t, \epsilon)]_{G_{\mu}} \\
\tilde{\xi}(t, \epsilon) & =\left[q(t) g_{\epsilon}(t), \omega(q(t, \epsilon))(\dot{q}(t, \epsilon))\right]_{G} \\
& =\left[q(t) g_{\epsilon}(t), \omega\left(q(t) g_{\epsilon}(t)\right)\left(T R_{g_{\epsilon}(t)}\left(\dot{q}(t)+\sigma_{q}\left(\dot{g}_{\epsilon} g_{\epsilon}^{-1}\right)\right)\right)\right]_{G} \\
& =\left[q(t), \omega(q(t))(\dot{q}(t))+\dot{g}_{\epsilon}(t) g_{\epsilon}^{-1}(t)\right]_{G}=\tilde{\xi}(t)+\left[q(t), \dot{g}_{\epsilon}(t) g_{\epsilon}^{-1}(t)\right]_{G}
\end{aligned}
$$

The typical structure equations of reduced vertical variations is obtained in the following way. Let $d / d \epsilon_{\epsilon=0} g_{\epsilon}(t)=\delta g(t) \in \mathfrak{g}$ and $\tilde{\eta}(t)=[q(t), \delta g(t)]_{G} \in \tilde{\mathfrak{g}}$. Using the definition of the covariant derivative on the associated bundle $\tilde{\mathfrak{g}} \rightarrow Q / G$, we may write

$$
\frac{D}{D t} \tilde{\eta}(t)=[q(t),[\omega(q(t))(\dot{q}(t)), \delta g(t)]]_{G}+[q(t), \dot{\delta g}(t)]_{G} .
$$

Finally, by computing the derivative to the curve $\epsilon \rightarrow \tilde{\xi}(t, \epsilon)$ we find the structure equation [2]:

$$
\tilde{\mathfrak{g}}_{x(t)} \ni \delta \tilde{\xi}(t):=\left.\frac{d}{d \epsilon}\right|_{0} \tilde{\xi}(t, \epsilon)=[q(t), \dot{\delta} g(t)]_{G}=\frac{D}{D t} \tilde{\eta}(t)-[\tilde{\xi}(t), \tilde{\eta}(t)] .
$$

It now remains to check the variation in the variable $y$. The curve $\epsilon \mapsto y(t, \epsilon)=[q(t, \epsilon)]_{G_{\mu}}$ is contained in a fibre of the bundle $\bar{\pi}_{\mu}^{-1}(x(t))$ and consequently, the tangent vector to this curve is in $V \bar{\pi}_{\mu}$. In the language of Proposition 10 the variation $\delta y$ in $y$ then corresponds to $(y, \tilde{\eta})+\tilde{\mathfrak{g}}_{\mu}$. 
Next, we describe the structure of reduced horizontal variations. Let $q(t, \epsilon)$ denote a deformation, with corresponding horizontal variation $\delta q(t)$. Again, the projection of the deformation $q(t, \epsilon)$ determines a deformation $(\dot{x}(t, \epsilon), y(t, \epsilon), \tilde{\xi}(t, \epsilon))$ of $(\dot{x}(t), y(t), \tilde{\xi}(t))$ in $T Q / G_{\mu}$ (with $x(t, \epsilon)=\pi(q(t, \epsilon)))$. By definition we have that $\tilde{\xi}(t, \epsilon)=[q(t, \epsilon), \omega(q(t, \epsilon))(\dot{q}(t, \epsilon))]_{G}$. Using the fact that $\delta q$ is horizontal, i.e. $\delta q=\delta x_{q}^{h}$, the equality

$$
\left.\frac{d}{d \epsilon}\right|_{0} \omega(q(t, \epsilon))(\dot{q}(t, \epsilon))=\Omega(q(t))(\delta q(t), \dot{q}(t))
$$

holds and we are able to write the horizontal and vertical parts of the tangent vector $\delta \tilde{\xi}(t) \in T_{\tilde{\xi}(t)} \tilde{\mathfrak{g}}$ as follows:

$$
\begin{aligned}
\delta \tilde{\xi}(t)^{v}=\left(\left.\frac{d}{d \epsilon}\right|_{\epsilon=0} \tilde{\xi}(t, \epsilon)\right)^{v} & =\left(\tilde{\xi}(t),[q(t), \Omega(q(t))(\delta q(t), \dot{q}(t))]_{G}\right) \\
& =(\tilde{\xi}(t), \tilde{\Omega}(x(t))(\delta x(t), \dot{x}(t))), \\
\delta \tilde{\xi}(t)^{h}=\left(\left.\frac{d}{d \epsilon}\right|_{\epsilon=0} \tilde{\xi}(t, \epsilon)\right)^{h} & =(\tilde{\xi}, \delta x(t)),
\end{aligned}
$$

where we used the decomposition of $T_{\tilde{\xi}(t)} \tilde{\mathfrak{g}}=\tilde{\pi}^{*} T(Q / G) \oplus V \tilde{\pi}$ into its horizontal and vertical subspace (due to the linear structure, $V \tilde{\pi}$ is identical to $\tilde{\mathfrak{g}} \times \tilde{\mathfrak{g}}$ ). It is important to see that $\delta \tilde{\xi}$ is not necessarily horizontal w.r.t the connection on the bundle $\tilde{\mathfrak{g}} \rightarrow Q / G$, despite the fact that it originates from a horizontal variation of $q(t)$.

The deformation $y(t, \epsilon)$ has a corresponding variation $\delta y(t)$ which is a tangent vector along $y(t)$. It is horizontal in terms of the connection associated to $\omega$ on the associated bundle $Q / G_{\mu} \rightarrow Q / G$.

\subsection{Lagrange-Poincaré reduction}

From the above structural equations for projected vertical and horizontal variations of a curve $q(t)$, we are now able to deduce the Lagrange-Poincaré equations on $T Q / G \cong T(Q / G) \times \tilde{\mathfrak{g}}$ for an invariant Lagrangian system $(Q, L, F)$ on $Q$. This follows easily from the following identity: given an arbitrary deformation of $q(t)$, then

$$
\int_{I} L(\dot{q}(t, \epsilon)) d t=\int_{I} l(\dot{x}(t, \epsilon), \tilde{\xi}(t, \epsilon)) d t .
$$

Now, if $q(t)$ is critical for $L$, then for any vertical variation with vanishing endpoints we write, with a slight abuse of notations

$$
\begin{aligned}
0 & =\frac{d}{d \epsilon} \int_{I} L(\dot{q}(t, \epsilon))=\frac{d}{d \epsilon} \int_{I} l(\dot{x}(t), \tilde{\xi}(t, \epsilon)) d t \\
& =\int_{I}\left\langle\mathbb{F}_{\tilde{\xi}} l(\dot{x}(t), \tilde{\xi}(t)), \frac{D}{D t} \tilde{\eta}(t)-[\tilde{\xi}(t), \tilde{\eta}(t)]\right\rangle d t \\
& =\int_{I}\left\langle-\frac{D}{D t} \mathbb{F}_{\tilde{\xi}} l-\operatorname{ad}_{\tilde{\xi}}^{*} \mathbb{F}_{\tilde{\xi}} l, \tilde{\eta}(t)\right\rangle d t,
\end{aligned}
$$

for all $\tilde{\eta}(t) \in \tilde{\mathfrak{g}}$ with vanishing endpoints. On the other hand if we consider horizontal variations, then

$$
\begin{aligned}
0 & =\frac{d}{d \epsilon} \int_{I} l(\dot{x}(t, \epsilon), \tilde{\xi}(t, \epsilon)) d t+\int_{I}\langle f(\dot{x}(t), \tilde{\xi}(t)), \delta x(t)\rangle d t \\
& \left.=\int_{I}\left(\left\langle\mathcal{E} \mathcal{L}(l)^{h}+f, \delta x\right\rangle+\left\langle\mathbb{F}_{\tilde{\xi}} l(\dot{x}, \tilde{\xi}), \tilde{\Omega}(x)(\delta x, \dot{x})\right)\right\rangle\right) d t
\end{aligned}
$$


Recall that $l$ can be regarded as an intrinsically constrained Lagrangian on $M=\tilde{\mathfrak{g}}$ (and $N=$ $Q / G)$, and hence $\mathcal{E} \mathcal{L}(l)$ in the previous equation is the Euler-Lagrange operator for $l$ regarded as a function on $T \tilde{\mathfrak{g}}$. The reduced equations now read

$$
\begin{aligned}
\frac{D}{D t} \mathbb{F}_{\tilde{\xi}} l & =-\operatorname{ad}_{\tilde{\xi}}^{*} \mathbb{F}_{\tilde{\xi}} l, \text { and } \\
\mathcal{E} \mathcal{L}(l)^{h} & =\left\langle\mathbb{F}_{\tilde{\xi}} l, i_{\dot{x}} \tilde{\Omega}\right\rangle-f .
\end{aligned}
$$

It should be clear that these equations are not the Euler-Lagrange equations for the intrinsically constrained Lagrangian system $(\tilde{\mathfrak{g}} \rightarrow Q / G, l, f)$ on the fibred manifold $\tilde{\mathfrak{g}} \rightarrow Q / G$. The introduction of the Routhian will avoid this obstacle, paying the price that additional non-conservative forces have to be taken into account.

\section{The Routhian reduction scheme}

Consider a critical curve $q(t), t \in[a, b]$, of the invariant Lagrangian system $(Q, L, F)$, that is

$$
0=\delta \int_{I} L(\dot{q}(t)) d t=-\int_{I}\langle F(\dot{q}(t)), \delta q(t)\rangle d t,
$$

with $\delta q(t)$ an arbitrary variation of $q(t)$. By considering variations with fixed endpoints, we obtain the Euler-Lagrange equations for critical curves

$$
\mathcal{E} \mathcal{L}(L)(\ddot{q}(t))=-F(\dot{q}(t)), \forall t \in I .
$$

We assume throughout this section that we fixed a principal connection $\omega$ and a regular momentum value $\mu \in \mathfrak{g}^{*}$ of $J_{L}$.

Definition 14 The Routhian is the function on $T Q$ defined by

$$
R^{\mu}\left(v_{q}\right)=L\left(v_{q}\right)-\left\langle\mu, \omega(q)\left(v_{q}\right)\right\rangle .
$$

The Routhian depends on the choice of the connection and the momentum $\mu$. Although the Routhian is not in general invariant under the action of $G$, we do have the property that the momentum map of the Routhian w.r.t the action of $G$ will be conserved (cf. proposition 4) . We thus study the behavior of the Routhian under the action of $G$.

Proposition 11 The Routhian is invariant under the action of $G_{\mu}$. It transforms under the infinitesimal action of $G$ as:

$$
\xi_{T Q}\left(R^{\mu}\right)\left(v_{q}\right)=\left\langle\operatorname{ad}_{\xi}^{*} \mu, \omega(q)\left(v_{q}\right)\right\rangle, \quad \forall \xi \in \mathfrak{g} .
$$

Proof. This is straightforward from the definition of $R^{\mu}$ and the equivariance of $\omega$.

\section{Proposition 12}

1. A critical curve of the invariant Lagrangian system $(Q, L, F)$ is a critical curve of the Lagrangian system $\left(Q, R^{\mu}, F+G^{\mu}\right)$ and vice versa, where $G^{\mu}$ is the gyroscopic force term

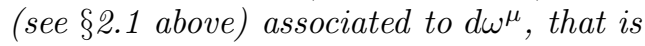

$$
G^{\mu}\left(v_{q}\right)=-i_{v_{q}} d \omega^{\mu}=-\left\langle\mu, i_{v_{q}} d \omega\right\rangle, \quad v_{q} \in T_{q} Q .
$$


2. The Lagrangian system $\left(Q, R^{\mu}, F+G^{\mu}\right)$ has conserved momentum $J_{R^{\mu}}$ w.r.t. the action of the entire symmetry group $G$.

3. The momentum maps of $L$ and $R^{\mu}$, both considered with respect to the full action of $G$, are related by $J_{R^{\mu}}=J_{L}-\mu$. Hence $q(t)$ is critical curve of the invariant Lagrangian system $(Q, L, F)$ with momentum $J_{L}=\mu$ if and only $q(t)$ is a critical curve of Lagrangian system $\left(Q, R^{\mu}, F+G^{\mu}\right)$ with momentum $J_{R^{\mu}}=0$.

Proof. The first statement is proven by considering the variation of $R^{\mu}$ along a critical curve $q(t)$ of $(Q, L, F)$ : given an arbitrary variation $\delta q(t)$ of $q(t)$ in $Q$ then

$$
\begin{aligned}
\delta \int_{I} R^{\mu}(\dot{q}(t)) d t & =\delta \int_{I} L(\dot{q}(t)) d t-\delta \int_{I}\left\langle\omega^{\mu}(q(t)), \dot{q}(t)\right\rangle d t \\
& =\delta \int_{I} L(\dot{q}(t)) d t+\int_{I}\left\langle i_{\dot{q}(t)} d \omega^{\mu}(q(t)), \delta q(t)\right\rangle d t-\left\langle\omega^{\mu}(q(t)), \delta q(t)\right\rangle_{a}^{b} \\
& =-\int_{I}\langle F(\dot{q}(t)), \delta q(t)\rangle d t-\int_{I}\left\langle G^{\mu}(\dot{q}(t)), \delta q(t)\right\rangle d t \\
& -\left\langle\omega^{\mu}(q(t)), \delta q\right\rangle_{a}^{b}+\left.\langle\mathbb{F} L(\dot{q}(t)), \delta q(t)\rangle\right|_{a} ^{b} .
\end{aligned}
$$

This easily proves the first statement in the proposition (the other direction follows by reversing the arguments).

For the second statement, according to Proposition 4, it is sufficient to show that $\xi_{T Q}\left(R^{\mu}\right)\left(v_{q}\right)=$ $-\left\langle F+G^{\mu}, \xi_{Q}\right\rangle(q)$. The contraction of the force term $F+G^{\mu}$ with vertical directions is precisely

$$
\begin{aligned}
\left\langle\left(F+G^{\mu}\right)\left(v_{q}\right), \xi_{Q}(q)\right\rangle & =\left\langle G^{\mu}\left(v_{q}\right), \xi_{Q}(q)\right\rangle \\
& =\left\langle\mu,\left[\omega(q)\left(v_{q}\right), \xi\right]\right\rangle \\
& =-\left\langle\operatorname{ad}_{\xi}^{*} \mu, \omega(q)\left(v_{q}\right)\right\rangle .
\end{aligned}
$$

Together with the previous proposition this shows the second statement.

Finally, the third statement is proven by computing the momentum map w.r.t action of $G$ of the Lagrangian system $\left(Q, R^{\mu}, F+G^{\mu}\right)$ :

$$
\begin{aligned}
\left\langle J_{R^{\mu}}\left(v_{q}\right), \xi\right\rangle & =\left.\frac{d}{d \epsilon}\right|_{\epsilon=0}\left(L\left(v_{q}+\epsilon \sigma_{q}(\xi)\right)-\left\langle\mu, \omega(q)\left(v_{q}+\epsilon \sigma_{q}(\xi)\right)\right\rangle\right) \\
& =\left\langle J_{L}\left(v_{q}\right)-\mu, \xi\right\rangle .
\end{aligned}
$$

We are now ready to state the first theorem of this paper. First we need to fix additional notations. The Routhian is reducible to a function

$$
\mathcal{R}^{\mu}: T Q / G_{\mu} \cong T(Q / G) \times Q / G_{\mu} \times \tilde{\mathfrak{g}} \rightarrow \mathbb{R} .
$$

Recall that the invariant force term $F$ reduces to a map

$$
f: T Q / G \rightarrow T^{*}(Q / G)
$$

and that the gyroscopic force term $G^{\mu}$ reduces to a gyroscopic force term

$$
\zeta^{\mu}: T\left(Q / G_{\mu}\right) \rightarrow T^{*}\left(Q / G_{\mu}\right)
$$


on $Q / G_{\mu}$ associated to $\beta^{\mu}$ (see Proposition 9). With these notations and those in 92 in mind, we now consider the bundle $M \rightarrow Q / G$, where

$$
M:=Q / G_{\mu} \times \tilde{\mathfrak{g}} \rightarrow Q / G .
$$

The triple $\left(M \rightarrow Q / G, \mathcal{R}^{\mu}, f+\zeta^{\mu}\right)$ will determine an intrinsically constrained Lagrangian system with

$$
T_{M} N=T(Q / G) \times Q / G_{\mu} \times \tilde{\mathfrak{g}} .
$$

To reduce the notational complexity, it is understood that $f$ and $\zeta^{\mu}$ are pull-backed to the appropriate bundles in order to fit the definition of an intrinsically constrained Lagrangian system.

Due to the product structure of the bundle $M=Q / G_{\mu} \times \tilde{\mathfrak{g}}$, we can restrict the Euler-Lagrange operator $\mathcal{E} \mathcal{L}\left(\mathcal{R}^{\mu}\right)$ to tangent vectors vertical to $M \rightarrow \tilde{\mathfrak{g}}$ or to $M \rightarrow Q / G_{\mu}$. The latter is denoted by $\partial_{y} \mathcal{R}^{\mu}$ and the first is precisely $\mathbb{F}_{\tilde{\xi}} \mathcal{R}^{\mu}$. The two bundles $Q / G_{\mu}$ and $\tilde{\mathfrak{g}}$ are equipped with a connection determined by $\omega$, and it is standard to show that these determine a unique connection on $M$ for which the horizontal distribution projects onto the horizontal distribution of $Q / G_{\mu}$ and $\tilde{\mathfrak{g}}$. It should be clear what is meant when we write $\mathcal{E} \mathcal{L}\left(\mathcal{R}^{\mu}\right)^{h}$ and $\mathcal{E} \mathcal{L}\left(\mathcal{R}^{\mu}\right)^{v}=\left(\partial_{y} \mathcal{R}^{\mu}, \mathbb{F}_{\tilde{\xi}} \mathcal{R}^{\mu}\right)$.

Theorem 1 (i) A critical curve of the invariant Lagrangian system $(Q, L, F)$ with momentum $\mu$ projects onto a critical curve of the intrinsically constrained Lagrangian system $(M \rightarrow$ $\left.Q / G, \mathcal{R}^{\mu}, f+\zeta^{\mu}\right)$. (ii) Conversely, if a critical curve of the intrinsically constrained Lagrangian system $\left(M \rightarrow Q / G, \mathcal{R}^{\mu}, f+\zeta^{\mu}\right)$ is the projection of a lifted curve $\dot{q}(t)$ in $T Q$, then $q(t)$ is a critical curve of $(Q, L, F)$ with momentum $\mu$.

Proof. The proof of this theorem is obtained by first considering the Lagrange-Poincaré reduction of the Lagrangian system $\left(Q, R^{\mu}, F+G^{\mu}\right)$ w.r.t the action of $G_{\mu}$, and consequently by showing that the reduced equations for critical curves with zero momentum coincide with the critical curves for the intrinsically constrained Lagrangian system $\left(M \rightarrow Q / G, \mathcal{R}^{\mu}, f+\zeta^{\mu}\right)$.

Let $q(t): I \rightarrow Q$ denote a critical curve of $(Q, L, F)$ with momentum $\mu$. From proposition 12 we know that $q(t)$ is a critical curve of the Lagrangian system $\left(Q, R^{\mu}, F+G^{\mu}\right)$ with momentum 0 .

The projection of $\dot{q}(t)$ onto $M$ is denoted by $(y(t), \tilde{\xi}(t))$. Recall that the vertical part of $\dot{y}(t)$ equals $(y(t), \tilde{\xi}(t))+\tilde{\mathfrak{g}}_{\mu}$ (this is the reduced version of the fact that the curve in $T Q$ is the complete lift of a curve in $Q$, see infra). By definition of $\mathcal{R}^{\mu}$, we may write $R^{\mu}(\dot{q}(t))=\mathcal{R}^{\mu}(\dot{x}(t), y(t), \tilde{\xi}(t))$, with $x(t)=\pi(q(t))$. We now show (i) by studying the reduction of the variational equation for critical curves of $\left(Q, R^{\mu}, F+G^{\mu}\right)$ with zero momentum (similar to section 3.5 for the Lagrangian system $(Q, L, F))$. Consider an arbitrary variation $\delta q(t)$ of $q(t)$ and the corresponding reduced variation $(\delta y(t), \delta \tilde{\xi}(t))$ of $(y(t), \tilde{\xi}(t))$, then (for notational convenience we omit the explicit time dependence)

$$
\begin{aligned}
\delta & \int_{I} R^{\mu}(\dot{q}) d t+\int_{I}\left\langle\left(F+G^{\mu}\right)(\dot{q}), \delta q\right\rangle d t \\
= & \delta \int_{I} \mathcal{R}^{\mu}(\dot{x}, y, \tilde{\xi}) d t+\int_{I}\left(\langle f(\dot{x}, \tilde{\xi}), \delta x\rangle+\left\langle\zeta^{\mu}(\dot{y}), \delta y\right\rangle\right) d t .
\end{aligned}
$$

Vertical Variations: We apply the Lagrange-Poincaré reduction scheme and we assume $\delta q(t)$ to be a vertical variation. From the previous section, we know that the reduced variations $\delta y(t)$ of $y(t)$ and $\delta \tilde{\xi}(t)$ of $\tilde{\xi}(t)$ both satisfy the following equalities:

$$
\begin{aligned}
\delta \tilde{\xi}(t) & =\frac{D}{D t} \tilde{\eta}(t)-[\tilde{\xi}(t), \tilde{\eta}(t)] \\
\delta y(t) & =(y, \tilde{\eta}(t))+\tilde{\mathfrak{g}}_{\mu} \\
\delta x(t) & =0
\end{aligned}
$$


where $\tilde{\eta}(t)$ can be chosen arbitrarily (with or without vanishing endpoints). From Eq. (8), we conclude that

$$
\delta \int_{I} \mathcal{R}^{\mu}(\dot{x}, y, \tilde{\xi}) d t+\int_{I}\left(\langle f(\dot{x}, \tilde{\xi}), \delta x\rangle+\left\langle\zeta^{\mu}(\dot{y}), \delta y\right\rangle\right) d t=0
$$

for reduced vertical variations of $(y(t), \tilde{\xi}(t))$. Moreover, since the variations are vertical w.r.t the fibration $M \rightarrow Q / G$, the first term is

$$
\delta \int_{I} \mathcal{R}^{\mu}(\dot{x}, y, \tilde{\xi}) d t=\int_{I}\left\langle\partial_{y} \mathcal{R}^{\mu}, \delta y\right\rangle+\left\langle\mathbb{F}_{\tilde{\xi}} \mathcal{R}^{\mu}, \delta \tilde{\xi}\right\rangle d t
$$

From the fact that $\mathbb{F}_{\tilde{\xi}} \mathcal{R}^{\mu}=\mathbb{F}_{\tilde{\xi}} l-\tilde{\mu}=0$, the variational equation holds for arbitrary vertical variations $(\delta y, \delta \tilde{\xi})$ of $(y, \tilde{\xi})$, and not only those satisfying $\delta \tilde{\xi}=\frac{D}{D t} \tilde{\eta}(t)-[\tilde{\xi}(t), \tilde{\eta}(t)]$. Therefore, the variational equation of the intrinsically constrained system $\left(M \rightarrow Q / G, \mathcal{R}^{\mu}, f+\zeta^{\mu}\right)$ is satisfied for arbitrary vertical variations in $(y(t), \tilde{\xi}(t))$ (i.e. vertical w.r.t the bundle $M \rightarrow Q / G$ ).

Horizontal variations. An arbitrary horizontal variation of $q(t)$ determines, after reduction to $M$, an arbitrary horizontal variation of $y(t)$ in $Q / G_{\mu}$. The corresponding variation of $\tilde{\xi}(t)$ is not horizontal w.r.t the associated connection on $\tilde{\mathfrak{g}}$ but the contribution of the vertical part to the variational equation will vanish since $\mathbb{F}_{\tilde{\xi}} \mathcal{R}^{\mu}=0$. We then have $\mathcal{E} \mathcal{L}\left(\mathcal{R}^{\mu}\right)^{h}=-\left(\left(\zeta^{\mu}\right)^{h}+f\right)$ which is

$$
\mathcal{E} \mathcal{L}\left(\mathcal{R}^{\mu}\right)^{h}=i_{\dot{x}} \tilde{\Omega}^{\mu}(y)-f(\dot{x}, \tilde{\xi})
$$

due to formula (5).

(ii) The converse statement is easily shown in the following way. Let $\dot{q}(t)$ denote a curve in $Q$ that projects onto $(\dot{x}(t), y(t), \tilde{\xi}(t))$, the latter curve being a a critical curve of the intrinsically constrained system on $Q / G_{\mu} \times \tilde{\mathfrak{g}}$. This implies that

$$
\delta \int_{I} \mathcal{R}^{\mu}(\dot{x}, y, \tilde{\xi}) d t+\int_{I}\langle f(\dot{x}, \tilde{\xi}), \delta x\rangle+\left\langle\zeta^{\mu}(\dot{y}), \delta y\right\rangle d t=0
$$

for an arbitrary variation of the critical curve. If we consider vertical variations to $(y(t), \tilde{\xi}(t))$ for which $\delta y(t)=0$, then $\mathbb{F}_{\tilde{\xi}} \mathcal{R}^{\mu}=j_{l}(\dot{x}, \tilde{\xi})-\tilde{\mu}(y)=0$. In terms of the curve upstairs, this is precisely $J_{L}(\dot{q}(t))=\mu$. Assume that we restrict the class of variations of $(y(t), \tilde{\xi}(t))$ to the projected variations of $q(t)$ living in the total space $Q$. By using the invariance of $R^{\mu}$, it is now easily seen from the previous variational equation for $\mathcal{R}^{\mu}$ that

$$
\delta \int_{I} R^{\mu}(\dot{q}) d t+\int_{I}\left\langle\left(F+G^{\mu}\right)(\dot{q}), \delta q\right\rangle d t=0
$$

Proposition 12 concludes the proof.

In the previous Theorem we introduced the reduction of a Lagrangian system $(Q, L, F)$ towards an intrinsically constrained Lagrangian system on a bundle $M$ over $Q / G$. It should be clear that due to the product structure of $M$, there are two intrinsic constraints: $\mathbb{F}_{\tilde{\xi}} \mathcal{R}^{\mu}=0$ or $j_{l}(\dot{x}, \tilde{\xi})=\tilde{\mu}(y)$ and $\partial_{y} \mathcal{R}^{\mu}+\zeta^{\mu}(\dot{y})^{v}=0$. The latter constraint belongs to the gyroscopic class (see section 2.1) and in the following Proposition we study this constraint in more detail.

Proposition 13 Let $\left(v_{x}, y, \tilde{\xi}\right)$ be arbitrary in $T_{M}(Q / G)=T(Q / G) \times Q / G_{\mu} \times \tilde{\mathfrak{g}}$ and $(y, \tilde{\eta})$ in $M=Q / G_{\mu} \times \tilde{\mathfrak{g}}$ with $\tilde{\eta}$ arbitrary, then

$$
\left\langle\partial_{y} \mathcal{R}^{\mu}\left(v_{x}, y, \tilde{\xi}\right),(y, \tilde{\eta})+\tilde{\mathfrak{g}}_{\mu}\right\rangle=-\left\langle\operatorname{ad}_{\tilde{\xi}}^{*} \tilde{\mu}(y), \tilde{\eta}\right\rangle
$$


Proof. The proposition follows from the following computations, with $\tilde{\eta}=[q, \dot{g}(0)] \in \tilde{\mathfrak{g}}$ arbitrary for some curve $g(\epsilon)$ in $G$ through the identity at $\epsilon=0$ :

$$
\begin{aligned}
\left\langle\partial_{y} \mathcal{R}^{\mu},(y, \tilde{\eta})+\tilde{\mathfrak{g}}_{\mu}\right\rangle & =-\left\langle\partial_{y}\langle\tilde{\mu}(y), \tilde{\xi}\rangle,(y, \tilde{\eta})+\tilde{\mathfrak{g}}_{\mu}\right\rangle \\
& =\left.\frac{d}{d \epsilon}\right|_{\epsilon=0}\left[q,\left\langle\mu, A d_{g(\epsilon)} \xi\right\rangle\right]=\left\langle\operatorname{ad}_{\tilde{\eta}}^{*} \tilde{\mu}, \tilde{\xi}\right\rangle=-\left\langle\operatorname{ad}_{\tilde{\xi}}^{*} \tilde{\mu}, \tilde{\eta}\right\rangle
\end{aligned}
$$

To provide a complete insight into the intrinsic constraint, we now compute $\left(\zeta^{\mu}\right)^{v}$. Let $v_{y} \in$ $T_{y}\left(Q / G_{\mu}\right)$ be arbitrary, then from formula (5) we find

$$
\left\langle\zeta^{\mu}\left(v_{y}\right),(y, \tilde{\eta})+\tilde{\mathfrak{g}}_{\mu}\right\rangle=-\beta^{\mu}\left(v_{y},(y, \tilde{\eta})+\tilde{\mathfrak{g}}_{\mu}\right)=\left\langle\operatorname{ad}_{v_{y}^{v}}^{*} \tilde{\mu}, \tilde{\eta}\right\rangle .
$$

The constraint on critical curves $(y(t), \tilde{\xi}(t))$ then reduces to $\operatorname{ad}_{\dot{y}^{v}(t)}^{*} \tilde{\mu}(y(t))=\operatorname{ad}_{\tilde{\xi}(t)}^{*} \tilde{\mu}(y(t))$. Due to the nondegeneracy of ad ${ }^{*}$, this is equivalent to saying that $\dot{y}^{v}(t)=(y(t), \tilde{\xi}(t))+\tilde{\mathfrak{g}}_{\mu}$.

We have thus proven the following reduction technique. Because it preserves the Lagrangian nature we refer to it as Routh reduction.

Theorem 2 Any critical curve $q(t)$ of an invariant Lagrangian system $(Q, L, F)$ with momentum $\mu$ projects onto a critical curve $(y(t), \tilde{\xi}(t))$ of the intrinsically constrained Lagrangian system $\left(M \rightarrow Q / G, \mathcal{R}^{\mu}, f+\zeta^{\mu}\right)$. The Euler-Lagrange equations for $\left(M \rightarrow Q / G, \mathcal{R}^{\mu}, f+\zeta^{\mu}\right)$ are

$$
\begin{aligned}
& \mathcal{E} \mathcal{L}\left(\mathcal{R}^{\mu}\right)^{h}(\ddot{x}, \dot{y}, \dot{\tilde{\xi}})=i_{\dot{x}} \tilde{\Omega}^{\mu}(y)-f(\dot{x}, \tilde{\xi}) \\
& j_{l}(\dot{x}, \tilde{\xi})=\tilde{\mu}(y) \\
& \dot{y}^{v}=(y, \tilde{\xi})+\tilde{\mathfrak{g}}_{\mu} .
\end{aligned}
$$

\subsection{Remarks on Routh reduction}

Remark 3 First we would like to draw the attention to the structure of the Routhian $R^{\mu}=$ $L-\omega^{\mu}$. If we start with a critical curve of the Lagrangian system $(Q, L, F)$ with momentum $\mu^{\prime} \neq \mu$ (that is, a critical curve of $\left(R^{\mu}, F-G^{\mu}\right)$ with non zero momentum), the projected curve $(y(t), \tilde{\xi}(t))$ is a critical curve of a variational problem for the system $\left(M \rightarrow Q / G, \mathcal{R}^{\mu}, f+\zeta^{\mu}\right)$ with constraints in the variations for $\tilde{\xi}$. The constraints are those described in Section 3.5 but w.r.t the action of $G_{\mu}$. Hence, $(y(t), \tilde{\xi}(t))$ may not be a critical solution of the free problem defined by $\left(M \rightarrow Q / G, \mathcal{R}^{\mu}, f+\zeta^{\mu}\right)$. The essential point is that, for momentum $\mu^{\prime}=\mu$, we have $\mathbb{F}_{\tilde{\xi}} \mathcal{R}^{\mu}=0$ along such projected critical curves; the constraint on $\delta \tilde{\xi}$ becomes negligible and the statement holds for arbitrary variations of $(y(t), \tilde{\xi}(t))$ in $M$. Moreover, the fact that $\mathbb{F}_{\tilde{\xi}} \mathcal{R}^{\mu}=0$ allows us to separate the variations in the variables $y$ and $\tilde{\xi}$. This is not possible if one works with the reduced lagrangian $l$. We could summarize this by saying that the Routhian $R^{\mu}$ is an alternative Lagrangian (up to gyroscopic forces) for $L$ such that critical curves with momentum $\mu$ become critical curves with zero momentum. Precisely this zero momentum condition will ensure that there are no constraints in the reduction of the variations.

Remark 4 Next, we wish to explicitly mention the case where $G$ is Abelian (for instance the case with cyclic coordinates). This assumption simplifies matters significantly. Firstly, we have that $G_{\mu}=G, \tilde{\mathfrak{g}}=\tilde{\mathfrak{g}}_{\mu}$. This implies that the map $\bar{\pi}_{\mu}$ is the identity and that the constraint 
$\dot{y}^{v}=(y, \tilde{\xi})+\tilde{\mathfrak{g}}_{\mu}$ in the Routhian reduced system $\left(M \rightarrow Q / G, \mathcal{R}^{\mu}, f+\zeta^{\mu}\right)$ is trivial. The equations of motion for this system then become:

$$
\begin{aligned}
& \mathcal{E} \mathcal{L}\left(\mathcal{R}^{\mu}\right)^{h}=i_{\dot{x}} \tilde{\Omega}^{\mu}(x)-f \\
& j_{l}(\dot{x}, \tilde{\xi})=\tilde{\mu}
\end{aligned}
$$

Remark 5 In order to relate the results presented here with the existing literature, we give an outline of what happens when the action of $G$ on $Q$ is a left action. The structure of the Routhian reduced equations remains the same, except that some objects will change sign due to the fact that the structure equation now takes the form $d \omega=\Omega+[\omega, \omega]$. This will affect the reduced objects in play, for instance the 2 -form $\beta^{\mu}$ becomes

$$
\beta^{\mu}=\left(\tilde{\Omega}^{\mu}, \operatorname{ad}^{*} \tilde{\mu}\right) .
$$

The Routhian reduced equations of motion are

$$
\begin{aligned}
& \mathcal{E} \mathcal{L}\left(\mathcal{R}^{\mu}\right)^{h}=i_{\dot{x}} \tilde{\Omega}^{\mu}(x)-f \\
& j_{l}(\dot{x}, \tilde{\xi})=\tilde{\mu}(y) \\
& \partial_{y} \mathcal{R}^{\mu}=\operatorname{ad}_{\dot{y}^{\nu}}^{*} \tilde{\mu}(y) .
\end{aligned}
$$

Note that also $\partial_{y} \mathcal{R}^{\mu}$ also changes sign in comparison with the right invariant case, so the third equation is equivalent to $\dot{y}^{v}=(y, \tilde{\xi})+\tilde{\mathfrak{g}}_{\mu}$.

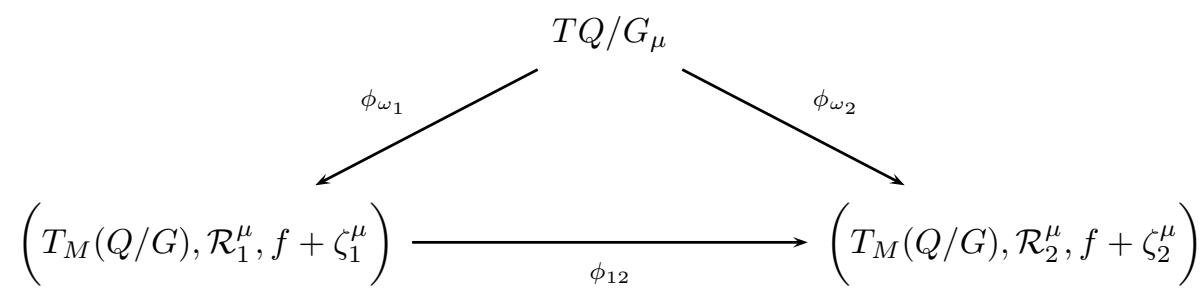

Figure 2: Different realizations of $T Q / G_{\mu}$.

Remark 6 The choice of the connection $\omega$ will alter the Routhian and force term in the reduced Lagrangian system in such a way that the critical curves of the intrinsically constrained Lagrangian systems coincide. Assume that two principal connections $\omega_{1}$ and $\omega_{2}$ are chosen. Then from standard connection theory both connections are equal up to an equivariant $\mathfrak{g}$-valued oneform $\delta$, i.e. $\omega_{2}=\omega_{1}+\delta$, with $R_{g}^{*} \delta=A d_{g^{-1}} \cdot \delta$ and $\langle\delta, \sigma(\xi)\rangle=0$ for all $\xi \in \mathfrak{g}$. The one-form $\delta$ reduces to a $\tilde{\mathfrak{g}}$-valued one-form on $Q / G$, denoted by $\tilde{\delta}$. Consider the one-form $\tilde{\delta}^{\mu}=\langle\tilde{\mu}, \tilde{\delta}\rangle$ on $Q / G_{\mu}$ (in fact it is a map from $Q / G_{\mu}$ to $T^{*}(Q / G)$ ). We now study the effect on the reduction process. First, the connections give two possible identifications $T Q / G_{\mu} \simeq T(Q / G) \times Q / G_{\mu} \times \tilde{\mathfrak{g}}$. The map $\phi_{12}$ (see Fig. 2) assumes the following form

$$
\phi_{12}\left(v_{x}, y, \tilde{\xi}\right)=\left(v_{x}, y, \tilde{\xi}+\tilde{\delta}\left(v_{x}\right)\right) .
$$

If we consider the Routhian functions $\mathcal{R}_{1}^{\mu}, \mathcal{R}_{2}^{\mu}$ and the force terms $\zeta_{1}^{\mu}, \zeta_{2}^{\mu}$ associated to $\omega_{1}$ and $\omega_{2}$ respectively, then

$$
\begin{aligned}
& \left(\phi_{12}^{*} \mathcal{R}_{2}^{\mu}\right)\left(v_{x}, y, \tilde{\xi}\right)=\mathcal{R}_{1}^{\mu}\left(v_{x}, y, \tilde{\xi}\right)-\left\langle\tilde{\delta}^{\mu}(y), v_{x}\right\rangle \\
& \zeta_{2}^{\mu}\left(v_{y}\right)=\zeta_{1}^{\mu}\left(v_{y}\right)+i_{v_{y}} d \tilde{\delta}^{\mu} .
\end{aligned}
$$


This shows that both (intrinsically constrained) Lagrangian systems are equivalent if we remember the fact that a linearly velocity dependent potential in a Lagrangian is equivalent to the addition of a gyroscopic force term (see [17]). The choice of the connection can be used to simplify the Routhian (paying the price that a gyroscopic force term is added to the system) or to simplify the gyroscopic force terms (paying the price that the Routhian will contain linear terms in the velocity). We return to this fact when we consider classical Routhian reduction and compare some recent geometric results in the literature and the formulation of the result as stated in [17.

Remark 7 We end this section with a presymplectic formulation of the Routh reduced system. Using the notations from Section 2.1 we can consider the pull-back of the canonical symplectic form $\omega_{Q / G}$ from $T^{*}(Q / G)$ to $T_{M}(Q / G)$ using the Legendre transform of $\mathcal{R}^{\mu}$. Since the intrinsic constraint associated to the fibration $Q / G_{\mu} \rightarrow Q / G$ comes from a regular gyroscopic term, the following theorem is a direct consequence of the definitions in Section 2.1. Here we use the projections $\bar{\pi}_{1}: T_{M}^{*}(Q / G) \rightarrow T^{*}(Q / G), \pi_{2}: M \rightarrow Q / G_{\mu}$.

Theorem 3 The Routhian reduced system of a Lagrangian system $(Q, L, F)$ can be formulated into a presymplectic system on $T Q / G_{\mu} \cong T_{M}(Q / G)$, where the presymplectic two-form is $\omega_{\mathcal{R}^{\mu}}=$ $\left(\bar{\pi}_{1} \circ \mathbb{F}_{1} \mathcal{R}^{\mu}\right)^{*} \omega_{Q / G}+\pi_{2}^{*} \beta^{\mu}$. The critical curves $(y(t), \tilde{\xi}(t))$ of the reduced system satisfy, with $\gamma(t)=(\dot{x}(t), y(t), \tilde{\xi}(t))$

$$
\left.\left(i_{\dot{\gamma}} \omega_{\mathcal{R}^{\mu}}=-d E_{\mathcal{R}^{\mu}}+f\right)\right|_{\gamma}
$$

In the case that $(Q, L, F)$ is conservative (i.e. $F=0)$, we can apply the constraint algorithm to show that there exists a submanifold $S$ of the final constraint submanifold on which a unique second-order vector field $\Gamma$ exists such that

$$
\left.\left(i_{\Gamma} \omega_{\mathcal{R}^{\mu}}=-d E_{\mathcal{R}^{\mu}}\right)\right|_{S}
$$

The integral curves of $\Gamma$ are lifted critical curves of the Routhian reduced system.

\section{Reconstruction}

The reconstruction process deals with the problem of finding a critical curve $q(t)$ of the Lagrangian system $(Q, L, F)$ such that $\dot{q}(t)$ projects onto a given critical curve $(y(t), \tilde{\xi}(t))$ of the intrinsically constrained Lagrangian system $\left(M \rightarrow Q / G, \mathcal{R}^{\mu}, f+\zeta^{\mu}\right)$ with $M=Q / G_{\mu} \times \tilde{\mathfrak{g}} \rightarrow Q / G$. Using Theorem 1, it is sufficient to construct a curve $q(t)$ in $Q$ that projects onto $(y(t), \tilde{\xi}(t))$ for all $t$, i.e. such that (i) $[q(t)]_{G_{\mu}}=y(t)$, and (ii) $\tilde{\xi}(t)=\left[q(t), \omega_{q(t)}(\dot{q}(t))\right]_{G}$.

To find such a curve $q(t)$ in $Q$, we choose an arbitrary starting point $q_{a} \in Q$ such that $\pi_{\mu}\left(q_{a}\right)=$ $y(a)$. Next, consider the unique horizontal curve $q_{h}(t)$ through $q_{a}$ that projects onto $x(t)$. If a curve $q(t)$ exists for which (i) and (ii) holds, then it is a gauge of $q_{h}(t)$, that is, $q(t)=q_{h}(t) g(t)$ for a certain curve $g(t)$ in $G$. The reconstruction process then only consists of solving a first order differential equation on $G$ to determine $g(t)$. For that purpose, let $\xi(t)$ be the curve in $\mathfrak{g}$ such that $\left[q_{h}(t), \xi(t)\right]_{G}=\tilde{\xi}(t) \in \tilde{\mathfrak{g}}$. Then, condition (ii) is satisfied if and only if $g(t)$ solves the first order differential equation

$$
\dot{g}(t) g^{-1}(t)=\xi(t),
$$

with $g(a)=e$. This determining condition for $g(t)$ comes from the fact that the projection of $\dot{q}(t)$ onto $\tilde{\mathfrak{g}}$ has to be $\tilde{\xi}(t)$. 
It now remains to check that (i) is true for the curve $q(t)=q_{h}(t) g(t)$, i.e. $y(t)=\left[q_{h}(t) g(t)\right]_{G_{\mu}}$. Fix a time $t$, then the horizontal part of the tangent vector to the curve $\left[q_{h}(t) g(t)\right]_{G_{\mu}}$ is the horizontal lift of $\dot{x}(t)$ to the point $y(t)$. Similarly it follows that the vertical component of the curve $\left[q_{h}(t) g(t)\right]_{G_{\mu}}$ is precisely $(y, \tilde{\xi})+\tilde{\mathfrak{g}}_{\mu}$. Since $g(a)=e$ the initial condition for $[q(t)]_{G_{\mu}}$ is $y(a)$, we may conclude that $y(t)$ and $[q(t)]_{G_{\mu}}$ coincide (both curves have the same horizontal and vertical parts and the same initial conditions). In this sense, the intrinsic constraint associated to the gyroscopic force $\zeta^{\mu}$ guarantees that $(y(t), \tilde{\xi}(t))$ is the projection of a lifted curve $\dot{q}(t)$ in $T Q$, i.e. it reflects the second-order nature of the original Lagrangian system $(Q, L, F)$. To conclude this section we unite Theorems 1,2 and 3

Theorem 4 Any critical curve of the Lagrangian system $(Q, L, F)$ on $Q$ with momentum $\mu$ projects in $M=Q / G_{\mu} \times \tilde{\mathfrak{g}}$ onto a critical curve of the intrinsically constrained Lagrangian system $\left(M \rightarrow Q / G, \mathcal{R}^{\mu}, f+\zeta^{\mu}\right)$. Conversely, any critical curve of $\left(M \rightarrow Q / G, \mathcal{R}^{\mu}, f+\zeta^{\mu}\right)$ is the projection of a critical curve of $(Q, L, F)$. The Euler-Lagrange equations of motion for the intrinsically constrained system are

$$
\begin{aligned}
& \mathcal{E} \mathcal{L}\left(\mathcal{R}^{\mu}\right)^{h}(\ddot{x}, \dot{y}, \dot{\tilde{\xi}})=i_{\dot{x}} \tilde{\Omega}^{\mu}(y)-f(\dot{x}, \tilde{\xi}) \\
& j_{l}(\dot{x}, \tilde{\xi})=\tilde{\mu}(y) \\
& \dot{y}^{v}=(y, \tilde{\xi})+\tilde{\mathfrak{g}}_{\mu} .
\end{aligned}
$$

These equations are equivalently formulated in a presymplectic setting. Let $\gamma(t)=(\dot{x}(t), y(t), \tilde{\xi}(t))$ in $T_{M}(Q / G)$ be associated to a curve $(y(t), \tilde{\xi}(t))$ in $M$. This curve is critical if and only if $\gamma(t)$ solves

$$
\left.\left(i_{\dot{\gamma}} \omega_{\mathcal{R}^{\mu}}=-d E_{\mathcal{R}^{\mu}}+f\right)\right|_{\gamma}
$$

\section{Routhian reduced systems: standard cases and examples}

\subsection{The regular case}

The classical geometric description of Routh reduction (for example 1], 15]) mainly differs from the one described above in that the unreduced Routhian is only defined in $J_{L}^{-1}(\mu)$ thus providing

a reduced Routhian independent of the variable $\tilde{\xi}$. This is done for $T-V$ Lagrangians. This classical setting is a special case of Theorem 4 when additional regularity conditions are assumed (the constraint $\mathbb{F} \mathcal{R}^{\mu}=0$ is a regular configuration constraint). The idea is, using the results from $₫ 2.2$, to eliminate the variable $\tilde{\xi}$ by means of the momentum equation $j_{l}\left(v_{x}, \tilde{\xi}\right)=\tilde{\mu}(y)$. For that purpose we now assume that the Lagrangian $L$ is both $G$-invariant and $G$-regular, i.e., the mapping $\mathbb{F}_{\tilde{\xi}} l$ is invertible (see 3.2 above). Let $\kappa_{l}: T(Q / G) \times \tilde{\mathfrak{g}}^{*} \rightarrow T(Q / G) \times \tilde{\mathfrak{g}}$ be the inverse of $\mathbb{F}_{\tilde{\xi}} l$.

There is a subtlety in the application of the result from $\$ 2.2$ Until now we have applied the results from \2 to Routh reduction by identifying $M$ with $Q / G_{\mu} \times \tilde{\mathfrak{g}}$ and $N$ with $Q / G$. In the following we deviate from this and we let $N$ be $Q / G_{\mu}$. In other words, we reinterpret the constrained system $\left(M \rightarrow Q / G, \mathcal{R}^{\mu}, f+\zeta^{\mu}\right)$ as a constrained system $\left(M \rightarrow Q / G_{\mu}, \mathcal{R}^{\mu}, f+\zeta^{\mu}\right)$, i.e. for now we forget the constraints in $y$ defined from the fact that $\mathcal{R}^{\mu}$ depends on $y$ but not on $\dot{y}$. Then, from $\oint 2.2$ it follows that the intrinsic constraint $\mathbb{F}_{\tilde{\xi}} \mathcal{R}^{\mu}=0$ is a regular configuration constraint, and using the inverse $\kappa_{l}$ we find a section $\gamma: T\left(Q / G_{\mu}\right) \rightarrow Q / G_{\mu} \times \tilde{\mathfrak{g}}$ in the sense of 2.2 given by

$$
\gamma\left(v_{y}\right)=\left(y, \kappa_{l}^{2}\left(v_{x}, \tilde{\mu}(y)\right)\right)
$$


where $v_{x}=T \bar{\pi}_{\mu}\left(v_{y}\right)$ with $\bar{\pi}_{\mu}: Q / G_{\mu} \rightarrow Q / G$ the natural projection; and $\kappa_{l}^{2}=\operatorname{pr}_{2} \circ \kappa_{l}$ with $\operatorname{pr}_{2}: T(Q / G) \times \tilde{\mathfrak{g}} \rightarrow \tilde{\mathfrak{g}}$ the projection onto the second factor. As in 2.2 , we are now able to consider the Lagrangian system on $N=Q / G_{\mu}$. However, recall that we temporarily forgot the intrinsic constraints in the variable $y$. We can reintroduce these constraints by making the remark that the resulting system on $N$ carries intrinsically constraints on the fibration $N=Q / G_{\mu} \rightarrow Q / G$. For notational convenience we directly introduce the Lagrangian system on $N$ as being a constrained system. This systems reads $\left(Q / G_{\mu} \rightarrow Q / G, \overline{\mathcal{R}}^{\mu}, \bar{f}+\zeta^{\mu}\right)$, where

$$
\begin{aligned}
& \overline{\mathcal{R}}^{\mu}: T_{Q / G_{\mu}}(Q / G) \rightarrow \mathbb{R}:\left(v_{x}, y\right) \mapsto \mathcal{R}^{\mu}\left(v_{x}, y, \kappa_{l}^{2}\left(v_{x}, \tilde{\mu}(y)\right)\right) \\
& \bar{f}: T_{Q / G_{\mu}}(Q / G) \rightarrow T^{*}(Q / G):\left(v_{x}, y\right) \mapsto f\left(\kappa_{l}\left(v_{x}, \tilde{\mu}(y)\right)\right)
\end{aligned}
$$

and $\zeta^{\mu}$ as usual the gyroscopic force associated to the 2 -form $\beta^{\mu}$ on $Q / G_{\mu}$. We conclude that the critical curves of the constrained Lagrangian system $\left(M \rightarrow Q / G, \mathcal{R}^{\mu}, f+\zeta^{\mu}\right)$ are in a one-to-one correspondence to the critical curves of $\left(Q / G_{\mu} \rightarrow Q / G, \overline{\mathcal{R}}^{\mu}, \bar{f}+\zeta^{\mu}\right)$.

Recall that $\beta^{\mu}$ can be decomposed into a horizontal and vertical part: $\left(\tilde{\Omega},-\mathrm{ad}^{*}\right)$. Similar to previous definitions, we let $\partial_{y} \overline{\mathcal{R}}^{\mu}$ be the shorthand notation for the restriction of the EulerLagrange operator $\mathcal{E} \mathcal{L}\left(\overline{\mathcal{R}}^{\mu}\right)$ to vectors vertical to $Q / G_{\mu} \rightarrow Q / G$. From Theorem 4 we easily have:

Theorem 5 Any critical curve of the Lagrangian system $(Q, L, F)$ with momentum $\mu$ projects in $Q / G_{\mu}$ onto a critical curve of the intrinsically constrained Lagrangian system $\left(Q / G_{\mu} \rightarrow\right.$ $\left.Q / G, \overline{\mathcal{R}}^{\mu}, \bar{f}+\zeta^{\mu}\right)$, and vice versa. The Euler-Lagrange equations of motion for the intrinsically constrained system are

$$
\begin{aligned}
& \mathcal{E} \mathcal{L}\left(\overline{\mathcal{R}}^{\mu}\right)^{h}(\ddot{x}, \dot{y})=i_{\dot{x}} \tilde{\Omega}^{\mu}(y)-\bar{f}(\dot{x}), \\
& \partial_{y} \overline{\mathcal{R}}^{\mu}(\dot{x}, y)=-\operatorname{ad}_{\dot{y}^{v}}^{*} \tilde{\mu}(y)
\end{aligned}
$$

The equation $\partial_{y} \overline{\mathcal{R}}^{\mu}(\dot{x}, y)=-\operatorname{ad}_{\dot{y}^{v}}^{*} \tilde{\mu}(y)$ can be rewritten as

$$
\dot{y}^{v}=\left(y, \kappa_{l}^{2}(\dot{x}, \tilde{\mu}(y))\right)+\tilde{\mathfrak{g}}_{\mu} .
$$

This theorem is similar to the classical geometric formulation of Routh reduction as in e.g. 9, 15] where the variational problem is confined to the set $J_{L}^{-1}(\mu)$. It is known that critical curves in $T Q$ with conserved momentum $\mu$ are in one-to-one correspondence to critical curves of the variational problem under the constraint $J_{L}^{-1}(\mu) \subset T Q$. In these references the Routhian is defined as the restriction of $R^{\mu}$ to $J_{L}^{-1}(\mu)$, and since this restriction $\left.R^{\mu}\right|_{J_{L}^{-1}(\mu)}$ is invariant with respect to the action of $G_{\mu}$, it is reducible to $J_{L}^{-1}(\mu) / G_{\mu}$. Using the fact that in the $G$-regular case (cf. Proposition 8)

$$
J_{L}^{-1}(\mu) / G_{\mu} \simeq T(Q / G) \times Q / G_{\mu},
$$

it is straightforward to show that this reduced Routhian is precisely $\overline{\mathcal{R}}^{\mu}$.

In [15] (as well as in the original work of Routh) the Lagrangian $L$ is of the type $L=T_{2}-V$ with $T_{2}=\frac{1}{2} \rho\left(v_{q}, v_{q}\right)$ a kinetic energy associated with a non-degenerate positively definite invariant metric $\rho$ on $Q$ and $V: Q \rightarrow \mathbb{R}$ an invariant potential energy. Following [2, 9] one chooses the principal connection to be the mechanical connection on $Q$ (the connection whose horizontal distribution is orthogonal to $V \pi$ w.r.t the kinetic energy metric $\rho$ ). The induced metric on $Q / G$ is denoted by $\bar{\rho}$ (defined by $\left.\bar{\rho}\left(v_{x}, v_{x}\right)=\rho\left(v_{x}^{h}, v_{x}^{h}\right)\right)$ and the vertical part of the metric defines a metric $\tilde{I}$ on $\tilde{\mathfrak{g}}$. This is called the inertia tensor and we assume it to be regular (i.e. the Lagrangian 
is $G$-regular). The momentum map then equals $j_{l}(x, \tilde{\xi})=b_{\tilde{I}}(x)(\tilde{\xi})=\tilde{\mu}(y)$ and the Routhian on $Q / G_{\mu}$ is

$$
\overline{\mathcal{R}}^{\mu}\left(v_{x}, y\right)=\frac{1}{2} \bar{\rho}\left(v_{x}, v_{x}\right)-\left(V(x)+\frac{1}{2}\langle\tilde{\mu}(y), \sharp \tilde{I}(x)(\tilde{\mu}(y))\rangle\right) .
$$

One should note that this Routhian may differ from the one found in classical books dealing with Routhian reduction. The difference lies in the fact that one typically works in a local coordinate chart, where the choice of the connection $\omega$ is taken to be the local connection with vanishing connection coefficients (and thus zero curvature). In the reduced system the curvature forceterm vanishes. This choice of connection with vanishing curvature will imply that the Routhian contains linear terms in the velocity, see also Remark [6]

For the reconstruction process in this regular situation one may proceed as in $\$ 5$ Indeed, given a solution $y(t)$ of the reduced problem defined by $\overline{\mathcal{R}}^{\mu}$ we consider the curve $\gamma(\dot{y}(t))$ in $Q / G_{\mu} \times \tilde{\mathfrak{g}}$, where $\gamma$ is as in (10). Then a family of critical curves $q(t)$ of the problem defined by $L$ can be recovered as in the general case. Alternatively (see [15, $\S I \mathrm{IV}]$ ), the curve $q(t)$ can be recovered by considering any curve $\bar{q}(t)$ with $y(t)=[\bar{q}(t)]_{G_{\mu}}$ and writing $q(t)=\bar{q}(t) g(t)$, where $g(t) \in G_{\mu}$ is obtained by requiring that $q(t) \in J_{L}^{-1}(\mu), \forall t$.

\subsubsection{The (pre)-symplectic nature of classical Routhian reduction}

It was formulated in [15] how the symplectic bundle picture for Routhian reduction looks like. Using Proposition 1 we can extend this to the more general case of presymplectic geometry. The Routhian reduced equations of motion in the classical case (so with a positive definite kinetic energy) are intrinsically constrained Euler-Lagrange equations living on $T_{Q / G_{\mu}}(Q / G)=$ $T(Q / G) \times Q / G_{\mu}$. Using the notations from Section 2 we have $M=Q / G_{\mu}, N=Q / G$. We can say that critical curves of the Routh reduced system are solutions to the following presymplectic system, with $\gamma(t)=(\dot{x}(t), y(t))$ :

$$
\left.\left(i_{\dot{\gamma}(t)}\left(\left(\bar{\pi}_{1} \circ \mathbb{F}_{1} \overline{\mathcal{R}}^{\mu}\right)^{*} \omega_{N}+\pi_{2}^{*} \beta^{\mu}\right)=-d E_{\overline{\mathcal{R}}^{\mu}}+\bar{f}\right)\right|_{\gamma}
$$

where $\mathbb{F}_{1} \overline{\mathcal{R}}^{\mu}: T(Q / G) \times Q / G_{\mu} \rightarrow T^{*}(Q / G) \times Q / G_{\mu}$ stands for the fiber derivative along the $T(Q / G)$ factor only. In the case that $\mathbb{F}_{1} \overline{\mathcal{R}}^{\mu}$ is a diffeomorphism then the presymplectic form is symplectic. This is the case if $L$ is a regular Lagrangian, see also [12].

\subsubsection{The modified Tippe Top}

The purpose of this example is to demonstrate the relevance of Routh reduction for nonconservative systems. In [3] a Tippe Top was described as a Lagrangian system with a dissipative force term. The system is invariant under the action of $S^{1}$ and a local stability analysis for relative equilibria was proven by means of Routh reduction. We only mention here the setup of this particular Lagrangian system within theoretical framework developed in this article. The configuration space is $Q=S O(3)$ and we will work in local coordinate chart determined by the Euler angles $(\varphi, \theta, \psi)$. We consider the Abelian group $G=S^{1}$ and the action on $S O(3)$ is defined by, for $\alpha \in S^{1}$ :

$$
(\varphi, \theta, \psi) \mapsto(\varphi+R \alpha, \theta, \psi-\epsilon \alpha),
$$

for $\epsilon, R>0$. Next, we define the Lagrangian $L$ which is of mechanical type and a dissipative force term $F$ which represents the friction due to the slipping of the contact point of the Tippe 
Top with the horizontal plane on which it evolves:

$$
\begin{aligned}
& L=\frac{1}{2}\left(\left(\epsilon^{2} m \sin ^{2} \theta+A\right) \dot{\theta}^{2}+A \sin ^{2} \theta \dot{\varphi}^{2}+C(\dot{\psi}+\dot{\varphi} \cos \theta)^{2}\right)-m g(R-\epsilon \cos \theta), \\
& F=-\mu(R-\epsilon \cos \theta)^{2} \dot{\theta} d \theta-\mu \epsilon \sin ^{2} \theta(\epsilon \dot{\varphi}+R \dot{\psi}) d \varphi-\mu R \sin ^{2} \theta(R \dot{\psi}+\epsilon \dot{\varphi}) d \psi,
\end{aligned}
$$

It is straightforward to see that $(Q, L, F)$ is invariant. The associated momentum map: $T Q \rightarrow \mathbb{R}$ is:

$$
\begin{aligned}
J_{L} & =\mathbb{F} L(q, \dot{q})\left(R \partial_{\phi}-\epsilon \partial_{\psi}\right)=R A \sin ^{2} \theta \dot{\varphi}+C(\dot{\psi}+\cos \theta \dot{\varphi})(R \cos \theta-\epsilon) \\
& =R\left(\left(A \sin ^{2} \theta+C \cos ^{2} \theta\right) \dot{\varphi}-C \epsilon \dot{\psi}\right)+C \cos \theta(R \dot{\psi}-\epsilon \dot{\varphi}),
\end{aligned}
$$

also called the Jellet integral. Fix a value $\mu \in \mathbb{R}$ of $J_{L}$. The group is abelian, therefore $G_{\mu}=S^{1}$, and $S O(3) / S^{1} \cong S^{2}$. In order to define the Routhian, we consider the following splitting of $T S O(3)$ : the horizontal subspace is defined by the (invariant) subspace spanned by

$$
\partial_{\theta} \text { and } \epsilon \partial_{\varphi}+R \partial_{\psi} \text {. }
$$

The curvature is zero, and we know that the Routh reduced system is a Lagrangian system on $S^{2}$, i.e. of the form $\mathcal{E} \mathcal{L}\left(\overline{\mathcal{R}}^{\mu}\right)=-\bar{f}$, cf. Theorem $[5$ with $\tilde{\Omega}=0$. In this case, the Routhian becomes:

$$
R^{\mu}=L-\mu\left(\frac{1}{\epsilon^{2}+R^{2}}(R \dot{\varphi}-\epsilon \dot{\psi})\right)
$$

The next step is to use the Jellet integral and to compute $\bar{R}^{\mu}$. Due to the complexity of the computations, we refer the reader to [3].

\subsubsection{The free rigid body}

We discuss the example of a free rigid body to illustrate the meaning of the equation $\partial_{y} \overline{\mathcal{R}}^{\mu}=$ $-\operatorname{ad}_{\dot{y}^{v}}^{*} \tilde{\mu}(y)$ in Theorem 5 We have shown that it corresponds to $\dot{y}^{v}=(y, \tilde{\xi})+\tilde{\mathfrak{g}}_{\mu}$, with $\tilde{\xi}$ determined from $j_{l}(\dot{x}, \tilde{\xi})=\tilde{\mu}(y)$ and here we illustrate this correspondence explicitly.

Following [15], the configuration space of the free rigid body is the entire group $Q=G=S O(3)$ on which $G$ acts from the left (in view of remark 5 we should change the sign of the 2 -form: $+\mathrm{ad}^{*}$ ). An element in $S O(3)$ is denoted by $A$ and corresponds to the rotation taking a reference configuration (with principle inertia axis) of the rigid body to its configuration at time $t$. The Lagrangian is the left invariant kinetic energy and the momentum equation corresponds to the spatial angular momentum. Using the notations from [15, we assume that the the fixed momentum equation corresponds to $\boldsymbol{\pi}=\mu \mathbf{k}$ (where $\boldsymbol{\pi}$ is the spatial angular momentum and $\mathbf{k}$ the unit vector in the positive $z$-axis). We choose the standard connection $\omega(A)(\dot{A})=\dot{A} A^{-1}$, and we identify the (trivial) bundle $\tilde{\mathfrak{g}}$ with $\mathfrak{g}$ by $[A, \xi]_{G} \mapsto A^{-1} \xi A$, i.e. the representant of $[A, \xi]_{G}$ at unity. The map determined by the connection $\omega$ which is used to identify $T S O(3) / G$ with $T(Q / G) \times \mathfrak{g} \cong \mathfrak{g}$ becomes $(A, \dot{A}) \mapsto A^{-1} \dot{A}$, i.e. the image is the angular velocity in the body reference frame. In a local coordinate neighborhood determined by Euler angles, the projection $T S O(3) \rightarrow \mathfrak{g} \cong \mathbb{R}^{3}$ equals

$$
(\phi, \theta, \psi, \dot{\phi}, \dot{\theta}, \dot{\psi}) \mapsto\left(\xi_{1}, \xi_{2}, \xi_{3}\right)^{T}
$$

with

$$
\begin{aligned}
& \xi_{1}=\dot{\theta} \cos \psi+\dot{\phi} \sin \theta \sin \psi \\
& \xi_{2}=-\dot{\theta} \sin \psi+\dot{\phi} \sin \theta \cos \psi \\
& \xi_{3}=\dot{\psi}+\dot{\phi} \cos \theta .
\end{aligned}
$$


The reduced Lagrangian $l$ then determines a function on $\mathfrak{g}$ and equals $l=\frac{1}{2}\left(I_{1} \xi_{1}^{2}+I_{2} \xi_{2}^{2}+I_{3} \xi_{3}^{2}\right)$, with $\left(I_{1}, I_{2}, I_{3}\right)$ the inertia tensor in the body reference frame.

The isotropy subgroup $G_{\mu}=S^{1}$ consists of the rotations about the k-axis; therefore $\pi_{\mu}: Q \rightarrow$ $Q / G_{\mu}=S^{2}: A \mapsto A^{-1} \mathbf{k}$. In terms of the Euler angles, this projection is $(\phi, \theta, \psi) \mapsto(\theta, \psi)$. The map $\tilde{\mu}: Q / G_{\mu} \rightarrow \tilde{\mathfrak{g}}^{*} \cong \mathfrak{g}^{*}$ is the momentum $\mu \mathbf{k}$ expressed in body coordinates:

$$
(\theta, \psi) \mapsto(\mu \sin \theta \sin \psi, \mu \sin \theta \cos \psi, \mu \cos \theta) .
$$

The configuration space of the Routh reduced system is simply $Q / G_{\mu}=S^{2}$ and the only meaningful equation from Theorem 5 is $\partial_{y} \overline{\mathcal{R}}^{\mu}=\operatorname{ad}_{\dot{y}^{v}}^{*} \tilde{\mu}(y)$.

In the following we will first compute the equation $\xi=\kappa_{l}^{2}(\tilde{\mu}(y))$ (see (10) ) and study its projection to the tangent bundle of $Q / G_{\mu}=S^{2}$. Secondly, we show that this projection corresponds to the equation $\partial_{y} \overline{\mathcal{R}}^{\mu}=\operatorname{ad}_{\dot{y}^{\nu}}^{*} \tilde{\mu}(y)$.

To compute $\kappa_{l}^{2}$ we start with the reduced momentum equation $j_{l}(\tilde{\xi})=\tilde{\mu}(y)$ :

$$
\begin{aligned}
I_{1} \xi_{1} & =\mu \sin \theta \sin \psi, \\
I_{2} \xi_{2} & =\mu \sin \theta \cos \psi, \\
I_{3} \xi_{3} & =\mu \cos \theta .
\end{aligned}
$$

It should be clear that since $I_{i} \neq 0$, we can compute $\xi$ explicitly

$$
\xi_{1}=\frac{1}{I_{1}} \mu \sin \theta \sin \psi, \xi_{2}=\frac{1}{I_{2}} \mu \sin \theta \cos \psi, \xi_{3}=\frac{1}{I_{3}} \mu \cos \theta
$$

Next, we need to compute the corresponding element in $T S^{2}$. In the general theory this is done by identifying $T S^{2}$ with the quotient $\bar{\pi}_{\mu}^{*} \tilde{\mathfrak{g}} / \tilde{\mathfrak{g}}_{\mu}$. In terms of the Euler-angles, this boils down to computing $\dot{\theta}$ and $\dot{\psi}$ out of (13): $\dot{\theta}=\xi_{1} \cos \psi-\xi_{2} \sin \psi$ and $\dot{\psi}=\xi_{3} \sin \theta-\left(\xi_{1} \sin \psi+\xi_{2} \cos \psi\right) \cos \theta$. If we substitute the values for $\xi_{1,2,3}$ in these two expressions, we find the equation $\dot{y}^{v}=(y, \tilde{\xi})+\tilde{\mathfrak{g}}_{\mu}$, with $\tilde{\xi}$ determined from $j_{l}(\tilde{\xi})=\tilde{\mu}(y)$ :

$$
\begin{aligned}
& \dot{\theta}=\mu \sin \theta \sin \psi \cos \psi\left(\frac{1}{I_{1}}-\frac{1}{I_{2}}\right), \\
& \dot{\psi}=\mu \cos \theta\left(\frac{1}{I_{3}}-\left(\frac{\sin ^{2} \psi}{I_{1}}+\frac{\cos ^{2} \psi}{I_{2}}\right)\right),
\end{aligned}
$$

The second way to retrieve these equations is using the Routhian $\overline{\mathcal{R}}^{\mu}$. By definition it is obtained from $\mathcal{R}^{\mu}=l(\xi)-\langle\tilde{\mu}(y), \xi\rangle$, where $\xi$ is substituted by (14):

$$
\overline{\mathcal{R}}^{\mu}(\theta, \psi)=-\frac{\mu^{2}}{2}\left(\frac{\sin ^{2} \theta \sin ^{2} \psi}{I_{1}}+\frac{\sin ^{2} \theta \cos ^{2} \psi}{I_{2}}+\frac{\cos ^{2} \theta}{I_{3}}\right) .
$$

Next, we compute the 2-form ad ${ }^{*}=\beta^{\mu}$ on $T S^{2}$. Recall that it is the projection of $d \omega^{\mu}$ to $Q / G_{\mu}$. In Euler angles, we have that $\langle\mu, \omega\rangle(A, \dot{A})=\tilde{\mu}(y) \cdot(\xi)^{T}=\mu(\dot{\phi}+\cos \theta \dot{\psi})$. Therefore $\beta^{\mu}=-\mu \sin \theta d \theta \wedge d \psi$, the gyroscopic force term is $\zeta^{\mu}=-i_{\dot{y}} \beta^{\mu}=-\mu \sin \theta \dot{\psi} d \theta+\mu \sin \theta \dot{\theta} d \psi$ and the Euler-Lagrange equations for the Routh reduced system are $\mathcal{E} \mathcal{L}\left(\overline{\mathcal{R}}^{\mu}\right)=-\zeta^{\mu}$ :

$$
\begin{aligned}
\frac{\partial \overline{\mathcal{R}}^{\mu}}{\partial \psi}=-\mu^{2} \sin ^{2} \theta \sin \psi \cos \psi\left(\frac{1}{I_{1}}-\frac{1}{I_{2}}\right) & =-\mu \sin \theta \dot{\theta} \\
\frac{\partial \overline{\mathcal{R}}^{\mu}}{\partial \theta}=-\mu^{2} \sin \theta \cos \theta\left(\left(\frac{\sin ^{2} \psi}{I_{1}}+\frac{\cos ^{2} \psi}{I_{2}}\right)-\frac{1}{I_{3}}\right) & =\mu \sin \theta \dot{\psi} .
\end{aligned}
$$

It should be clear that these Euler-Lagrange equations, when brought into normal form, are precisely the equations of motion obtained above by projecting $\tilde{\xi}=\kappa_{l}^{2}(\tilde{\mu}(y))$ to $T S^{2}$. 


\subsection{Lagrangian systems subjected to magnetic forces}

Assume that we study a conservative Lagrangian system $L$, where $L=T_{2}+T_{1}-V, T_{2}=\frac{1}{2} \rho\left(v_{q}, v_{q}\right)$ is the kinetic energy and $T_{1}=\left\langle\alpha(q), v_{q}\right\rangle$ with $\alpha$ and invariant 1-form. These linear terms in the kinetic energy are found, for example, when the mechanical system is subject to magnetic force terms (for instance, charged particle/rigid body evolving in a magnetic field). The restriction of $\alpha$ to horizontal vectors is denoted by $\alpha^{h}$ and is projectable to a one-form $\bar{\alpha}$ on $Q / G$. The restriction of $\alpha$ to verticals determines a section $\tilde{\alpha}^{v}$ of $\tilde{\mathfrak{g}}^{*} \rightarrow Q / G$. We consider the mechanical connection $\omega$ defined by the metric $\rho$ as before, and let $\tilde{I}$ be a regular inertia-tensor (i.e. $L$ is $G$-regular). The momentum condition then reads

$$
b_{\tilde{I}} \tilde{\xi}+\tilde{\alpha}^{v}=\tilde{\mu},
$$

with inverse $\tilde{\xi}=\sharp_{\tilde{I}}\left(\tilde{\mu}-\tilde{\alpha}^{v}\right)$. The Routhian on $Q / G_{\mu}$ assumes the form

$$
\overline{\mathcal{R}}^{\mu}\left(v_{x}, y\right)=\frac{1}{2} \bar{\rho}\left(v_{x}, v_{x}\right)+\left\langle\bar{\alpha}(x), v_{x}\right\rangle-\left(V(x)+\frac{1}{2}\left\langle\left(\tilde{\mu}-\tilde{\alpha}^{v}\right), \sharp \tilde{I}\left(\tilde{\mu}-\tilde{\alpha}^{v}\right)\right\rangle\right) .
$$

\subsubsection{The heavy top in a magnetic field}

We consider a constant magnetic field along the $z$-axis in the inertia frame. The Lagrangian of a charged rigid body with a fixed point is invariant under the left action of rotations about the $z$-axis (i.e. when $(\phi, \theta, \psi)$ is a coordinate chart determined by the Euler-angles, then $\phi$ is (locally) cyclic). Thus $Q=S O(3), G_{\mu}=G=S^{1}$ and $Q / G=S^{2}$, see also [15]. The Lagrangian equals, with $\xi_{i}$ as written down in Eq. (12):

$$
L=\frac{1}{2} \sum_{i=1}^{3} I_{i} \xi_{i}-m g A^{-1} \mathbf{k} \cdot \boldsymbol{\chi}-\Omega B A^{-1} \mathbf{k} \cdot I \boldsymbol{\xi},
$$

with $\Omega=q / m c$, with $q$ the charge of the heavy top, $g$ the earth's acceleration, $m$ the mass and $\chi$ the vector with length $\epsilon$ connecting the fixed point with the center of mass in the body reference frame. We assume that the top is symmetric and that the moving frame is directed along its symmetry axis (i.e. $I_{1}=I_{2}$ ). In this case, the Lagrangian becomes

$$
L=\frac{1}{2} I_{1} \dot{\theta}^{2}+\frac{1}{2} I_{3} \dot{\psi}^{2}+I_{3} \cos \theta \dot{\phi} \dot{\psi}+\frac{1}{2} \rho_{\phi \phi} \dot{\phi}^{2}-m g \epsilon \cos \theta-\Omega B\left(\rho_{\phi \phi} \dot{\phi}+I_{3} \cos \theta \dot{\psi}\right),
$$

with $\rho_{\phi \phi}=I_{1} \sin ^{2} \theta+I_{3} \cos ^{2} \theta$. Thus $\alpha=-\Omega B\left(\rho_{\phi \phi} d \phi+I_{3} \cos \theta d \psi\right), \tilde{\alpha}^{v}=-\Omega B \rho_{\phi \phi}$ and $\alpha^{h}=0$. Here we have chosen $\omega$ to be the mechanical connection $\omega=d \phi+\left(I_{3} \cos \theta d \psi\right) / \rho_{\phi \phi}$. The conserved momentum $J_{L}$ reads

$$
\rho_{\phi \phi} \dot{\phi}+I_{3} \cos \theta \dot{\psi}-\Omega B \rho_{\phi \phi}=\mu .
$$

The next step is the computation of the Routhian $\overline{\mathcal{R}}^{\mu}$, which can be carried out in two ways: either one computes $\dot{\phi}$ out of the momentum equation, and then substitute the expression in $L-\mu\left(\dot{\phi}+\left(I_{3} \cos \theta \dot{\psi}\right) / \rho_{\phi \phi}\right)$, or one computes $\bar{\rho}$ and use the expression of $\overline{\mathcal{R}}^{\mu}$ determined above. After straightforward but tedious computations one finds that the Routhian $\overline{\mathcal{R}}^{\mu}$ for the magnetic heavy top assumes the following form

$$
\overline{\mathcal{R}}^{\mu}=\frac{1}{2}\left(I_{1} \dot{\theta}^{2}+\frac{I_{3} I_{1} \sin ^{2} \theta}{\rho_{\phi \phi}} \dot{\psi}^{2}\right)-V_{\mu}(\theta)
$$


with

$$
V_{\mu}(\theta)=m g \cos \theta+\frac{1}{2} \frac{\left(\mu+\Omega B \rho_{\phi \phi}\right)^{2}}{\rho_{\phi \phi}} .
$$

In the case that $\Omega B \ll 1$, we may neglect the term in $(\Omega B)^{2}$ and then the effective potential $V_{\mu}$ equals up to constant the effective potential for the heavy top (i.e. set $B=0$ ). In this case the critical curves of both problems coincide, but the reconstruction process will differ. The reconstruction equation, roughly said, is the inverse of the momentum map:

$$
\dot{\phi}=\frac{\mu-I_{3} \cos \theta \dot{\psi}}{\rho_{\phi \phi}}+\Omega B
$$

This result is also known as Larmor's theorem (for example, see [5]): the motion of a charged particle in a constant magnetic field is the motion of the particle in the absence of the magnetic field superposed with a rotation with constant frequency $\Omega B$, the Larmor frequency.

\subsection{Lagrangians linear in the symmetry generators}

Here we study the case where the Lagrangian of an invariant conservative Lagrangian system $(Q, L, 0)$ generates a reduced momentum map $j_{l}$ that does not depend on the variable $\tilde{\xi}$, i.e. for a critical curve $(y(t), \tilde{\xi}(t))$ projecting onto $x(t)$ :

$$
j_{l}(\dot{x})=\tilde{\mu}(y) .
$$

This situation occurs for instance if one studies Lagrangian systems for which the kinetic energy is not strictly positive definite, i.e. if $L\left(v_{q}\right)=\frac{1}{2} \rho(q)\left(v_{q}, v_{q}\right)-V(q)$, with $\rho$ and $V$ right invariant, $\rho$ a symmetric two-tensor such that $\rho(\sigma(\xi), \sigma(\xi)) \equiv 0$ for any $\xi \in \mathfrak{g}$.

In this situation we can consider the metric $\bar{\rho}$ on $Q / G$ defined in the usual way as $\bar{\rho}\left(v_{x}, v_{x}\right)=$ $\rho(q)\left(\left(v_{x}\right)_{q}^{h},\left(v_{x}\right)_{q}^{h}\right)$, for $\pi(q)=x$ and $v_{x}$ arbitrary in $T_{x}(Q / G)$. The remaining part of $\rho$ yields a $\tilde{\mathfrak{g}}^{*}$-valued one-form $\tilde{\rho}$ on the quotient space $Q / G$. The momentum constraint for a critical curve then yields $\tilde{\rho}(x)(\dot{x})=\tilde{\mu}(y)$.

We can apply Theorem 2 and we find that the Routhian $\mathcal{R}^{\mu}\left(v_{x}, y, \tilde{\xi}\right)$ equals

$$
\frac{1}{2} \bar{\rho}(x)\left(v_{x}, v_{x}\right)-V(x)+\langle(\tilde{\rho}(x)-\tilde{\mu}(y)), \tilde{\xi}\rangle .
$$

From Section 2, Proposition 2 we know that the critical curves of this intrinsically constrained Lagrangian system $\left(M \rightarrow Q / G, \mathcal{R}^{\mu}, \zeta^{\mu}\right)$ project onto critical curves of the Lagrangian system $\left(Q / G_{\mu}, \overline{\mathcal{R}}^{\mu}, \zeta^{\mu}\right)$ constrained to the submanifold in $T\left(Q / G_{\mu}\right)$ determined by $\tilde{\rho}(x)\left(v_{x}\right)=\tilde{\mu}(y)$. The function $\overline{\mathcal{R}}^{\mu}$ is defined as

$$
\overline{\mathcal{R}}^{\mu}\left(v_{x}\right)=\frac{1}{2} \bar{\rho}(x)\left(v_{x}, v_{x}\right)-V(x),
$$

where we identified $V$ with its reduced function on $Q / G$. This particular situation is encountered in general relativity, where solutions to Einstein's equations admit lightlike Killing vectors.

\subsubsection{Lightlike Killing vectors}

This example shows how the presented theory can be applied to reduce geodesic equations in general relativity where the metric admits a lightlike Killing vector. We assume that the Killing vector $X$ is complete, i.e. the flow of $X$ determines an Abelian group action of $\mathbb{R}$ on the spacetime 
$(M, g)$. Since $X$ is Killing it determines a symmetry of the kinetic energy Lagrangian $L\left(v_{q}\right)=$ $\frac{1}{2} g\left(v_{q}, v_{q}\right)$.

A relevant local example from general relativity illustrating the above can be found in the so called pp-wave solution to Einstein's equations (see [11]):

$$
g=H(u, x, y) d u^{2}+2 d u d v+d x^{2}+d y^{2},
$$

expressed in the Brinckmann coordinates. The vector field $\partial_{v}$ is Killing, and the momentum constraint then assumes the form $J_{L}(u, x, y, \dot{u})=\dot{u}=\mu$. The geodesics with momentum $\mu$ are critical curves for the variational system on $T \mathbb{R}^{3}$ with Lagrangian equal to

$$
\overline{\mathcal{R}}^{\mu}=\frac{1}{2}\left(\mu^{2} H(u, x, y)+\dot{x}^{2}+\dot{y}^{2}\right)
$$

constrained to the submanifold $\dot{u}=\mu$.

\section{Acknowledgements}

The authors want to thank Tudor S. Ratiu for useful conversations about some examples where the classical approach can not be carried out. We are also indebted to Frans Cantrijn for the careful reading of this paper. MCL was partially supported by Ministerio de Ciencia y Tecnología of Spain under grants MTM2007-60017 and MTM2008-01386 and BL by a Research Programme of the Research Foundation - Flanders (FWO).

\section{References}

[1] V.I. Arnold. Dynamical Systems III, volume Encyclopaedia of Mathematics. Springer-Verlag, 1988.

[2] H. Cendra, J.E. Marsden, and T.S. Ratiu. Lagrangian Reduction by Stages, volume 152 of Memoirs of the American Mathematical Society. 2001.

[3] M.C. Ciocci and B. Langerock. Dynamics of the Tippe Top via Routhian Reduction. Regul. and Chaotic Dyn., 12:602-614, 2007.

[4] M. Crampin and T. Mestdag. Routh's procedure for non-Abelian symmetry groups. J. Math. Phys., 49, 2008.

[5] H. Goldstein. Classical Mechanics. Addison-Wesley Series in Physics. Addison-Wesley Publishing Co., Reading Mass., second edition, 1980.

[6] M.J. Gotay and J.M. Nester. Presymplectic Lagrangian systems I: the constraint algorithm and the euivalence problem. Ann. Inst. Henri Poincaré, 30(2):129-142, 1979.

[7] M.J. Gotay and J.M. Nester. Presymplectic Lagrangian systems II: the second-order equation problem. Ann. Inst. Henri Poincaré, 32(1):1-13, 1980.

[8] M.J. Gotay, J.M. Nester, and G. Hinds. Presymplectic manifold and the Dirac-Bergmann theory of constraints. J. Math. Phys., 19(11):2388-2399, 1978. 
[9] S.M. Jalnapurkar and J.E. Marsden. Reduction of Hamilton's variational principle. Dynamics and Stability of Systems, 15(3):287-318, 2000.

[10] S. Kobayashi and K. Nomizu. Foundations of differential geometry, volume I and II. Intersience Publishers, 1963.

[11] D. Kramer, H. Stephani, E. Herlt, and M. MacCallum. Exact solutions of Einstein's field equations. Number 6 in Cambridge monographs on mathematical physics. Cambridge University Press, 1980.

[12] B. Langerock, F. Cantrijn, and J. Vankerschaver. Routhian reduction for quasi-invariant Lagrangians. J. Math. Phys., 51(2), 2010. math.DG/09120863.

[13] D. Lewis. Lagrangian block diagonalization. J. Dynamics and diff. Equations, 4(1), 1992.

[14] M. Castrillón López and T.S. Ratiu. Reduction in principal bundles: Covariant LagrangePoincaré equations. Comm. Math. Phys., 236:223-250, 2003.

[15] J.E. Marsden, T.S. Ratiu, and J. Scheurle. Reduction theory and the Lagrange-Routh equations. Journal of Mathematical Physics, 41(6):3379-3429, 2000.

[16] Kenneth R. Meyer. Symmetries and integrals in mechanics. In Dynamical systems (Proc. Sympos., Univ. Bahia, Salvador, 1971), pages 259-272. Academic Press, New York, 1973.

[17] L.A. Pars. A Treatise on Analytical Dynamics. Heinemann Educational Books, 1965. 